\title{
GEOMETRY OF THE SEVERI VARIETY
}

\author{
STEVEN DIAZ AND JOE HARRIS
}

\begin{abstract}
This paper is concerned with the geometry of the Severi variety $W$ parametrizing plane curves of given degree and genus, and specifically with the relations among various divisor classes on $W$. Two types of divisor classes on $W$ are described: those that come from the intrinsic geometry of the curves parametrized, and those characterized by extrinsic properties such as the presence of cusps, tacnodes, hyperflexes, etc. The goal of the paper is to express the classes of the extrinsically defined divisors in terms of the intrinsic ones; this, along with other calculations such as the determination of the canonical class of $W$, is carried out by using various enumerative techniques. One corollary is that the variety of nodal curves of given degree and genus in the plane is affine.
\end{abstract}

1. Introduction and statements. The basic objects of study here will be families of plane curves of a given degree and genus, and the first order of business is to define the varieties we will be looking at.

(a) Severi varieties. The set of all plane curves of a given degree $d$ is of course parametrized by a projective space $\mathbf{P}^{N}$. What is of interest to us here is not the space $\mathbf{P}^{N}$ itself, but the various subvarieties of $\mathbf{P}^{N}$ characterized by the geometry of the curves they parametrize. The most general problem along these lines would be to describe completely the geometry of the equisingular stratification of $\mathbf{P}^{N}$, that is, the loci of curves of a given topological type and how these loci fit together. This problem seems to be well beyond our reach at present, since even the local deformation spaces of plane curve singularities may be very mysterious. A coarser, but still very natural, stratification of $\mathbf{P}^{N}$ (or at least the open subset of reduced curves) is simply by geometric genus; we may hope to describe the geometry of the locus $V_{d, g}$ of reduced curves of given geometric genus. Even here, however, the local picture is already very cloudy, sending us to the further simplification of the variety $V=V^{d, \delta}$ of nodal curves of degree $d$ and geometric genus $g$, that is, reduced and irreducible curves of degree $d$ having exactly $\delta=\frac{1}{2}(d-1)(d-2)-g$ nodes as singularities (see [Fulton] for a discussion).

Happily, the subvariety $V \subset \mathbf{P}^{N}$ is very well behaved: it is irreducible and smooth of dimension $N-\delta$, and there exists a universal family of smooth curves of genus $g$ mapping onto it. Unhappily, we may have cut away too much: the problem is that $V$ is not complete-its divisor theory, for example, is, conjecturally, trivial (see below) - and before we can really work with it we have to remedy, in part at least, this defect. The first basic problem, then, is to find a good compactification

Received by the editors April 13, 1987.

1980 Mathematics Subject Classification (1985 Revision). Primary 14H10; Secondary 14C22, $14 \mathrm{H} 99$.

First author supported by NSF Postdoctoral Research Fellowship. Second author supported by NSF grant number DMS-84-02209. 
$W$ of $V$. Here by "good" we mean essentially that the points of $W$ should actually correspond to geometric objects (i.e., $W$ should represent a geometrically defined functor), and at the same time the geometry of $W$ should be tractable: for example, its singularities should be describable, and not too bad.

An obvious thing to try is just to take the closure of $V$ in the space $\mathbf{P}^{N}$. This, however, fails both of the criteria above: we do not know what plane curves are limits of nodal curves of given degree and genus; and, worse, the singularities of the closure of $V$ are unspeakable. Another possibility is to put some additional structure on the plane curve: for example, consider the natural map of $V$ to the moduli space $\overline{\mathscr{M}}_{g}$ of stable curves of genus $g$, or the map of $V$ to the Hilbert scheme $\mathscr{H}$ of zero-dimensional subschemes of $\mathbf{P}^{2}$ of degree $\delta$ assigning to every curve $C \in V$ its nodes, or the map of $V$ to the projective space $\mathbf{P}^{M}$ of curves of degree $2 d+2 g-2$ associating to each $C \in V$ its dual curve; and in each case take the closure of the graph of the map. All of these are potentially better compactifications than the naive closure of $V$ in $\mathbf{P}^{N}$ (in particular, on the basis of investigations by the first author of this paper, the second approach looks promising; while it may be hoped that work such as Ziv Ran's [Ran] might shed some light on the first). To date, however, none has been completely worked out.

Our (compromise) solution in this paper has been to work with a partial closure of $V$ : we will look first at the union $\tilde{V}$ of $V$ with all the codimension 1 equisingular strata in the closure $\bar{V}$. This has the virtue that (as we will see) the local geometry of $\tilde{V}$ is clearly understood, and also we can say exactly which curves $C \in \mathbf{P}^{N}$ lie in $\tilde{V}$. Moreover, while $\tilde{V}$ is not complete, it is the complement of a codimension 2 subvariety in a projective variety, so that, e.g., if we describe the divisor theory of $\tilde{V}$ we will know it on any reasonable compactification of $V$.

Let us now describe the variety $\tilde{V}$. From the semistable reduction theorem for families of curves, it follows that for any curve $C \in \bar{V}$ the sum of the geometric genera of the components of $C$ (with their reduced structures) will be at most $g$. This, together with standard dimension counts, shows that the locus of nonreduced curves in the closure $\bar{V}$ of $V$ has codimension strictly greater than one. With this and the results of [Diaz-Harris], we see that $\tilde{V}$ consists of the union of $V$ with

the locus $C U$ of reduced and irreducible curves of genus $g$ with $\delta-1$ nodes and one cusp;

the locus $T N$ of reduced and irreducible curves of genus $g$ with $\delta-2$ nodes and one tacnode;

the locus $T R$ of reduced and irreducible curves of genus $g$ with $\delta-3$ nodes and one ordinary triple point; and

the locus $\Delta$ of reduced curves of geometric genus $g-1$, having at most two irreducible components, with $\delta+1$ nodes.

Each of these loci is of pure codimension one in $\tilde{V}$. The first three we suspect, but do not know, to be irreducible; the locus $\Delta$ is the union of the component $\Delta_{0}$ of reduced and irreducible nodal curves of genus $g-1$, and various components consisting of reducible curves, as we will see below. Moreover, we know that $\tilde{V}$ looks like in a neighborhood of each of these loci: by standard deformation theory (see for example [Diaz-Harris]), given a curve $C$ in any of these loci and any singular point $p$ of $C$, the space $U$ of deformations of $C$ preserving the singularities of $C$ other than $p$ maps with surjective differential onto the étale versal deformation 
space $B$ of the singularity at $p$; the variety $\tilde{V}$ will then be the inverse image of the equigeneric locus in $B$. Thus, for example, the étale versal deformation space of the cusp $y^{2}=x^{3}$ is given by

$$
y^{2}=x^{3}+a x+b
$$

and the equigeneric locus-in this case just the locus of $(a, b)$ for which the fiber is singular-is given by the discriminant $4 a^{3}+27 b^{2}$. The variety $\tilde{V}$ thus looks, in a neighborhood of $C U$, like the product of a cuspidal curve and a smooth $(N-\delta-1)$ dimensional variety (Figure 1 ).

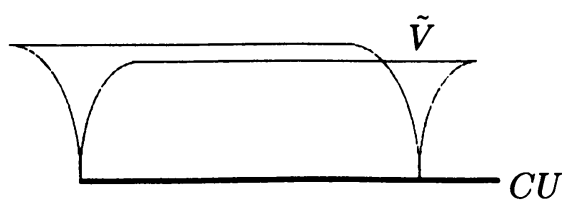

FIGURE 1

Similarly, the deformation space of the tacnode $y^{2}=x^{4}$ is given by

$$
y^{2}=x^{4}+a x^{2}+b x+c ;
$$

and the equigeneric locus--the locus of $(a, b, c)$ for which the corresponding fiber has two nodes-is the smooth curve with equations $b=0, a^{2}=4 c$. The variety $\tilde{V}$ is thus smooth in a neighborhood of the locus $T N$; and a similar calculation shows that $\tilde{V}$ is also smooth near $T R$. Finally, we see that, in a neighborhood of a point $C$ of $\Delta, \tilde{V}$ is the union of smooth sheets corresponding to nodes of the curve $C$; see Figure 2. (If $C$ is irreducible, there are $\delta+1$ sheets, corresponding to all the nodes of $C$; if $C$ is the union of components $C_{1}$ and $C_{2}$ of degrees $d_{1}$ and $d_{2}$ there will be $d_{1} d_{2}$ sheets, corresponding to the points of intersection of $C_{1}$ and $C_{2}$.)

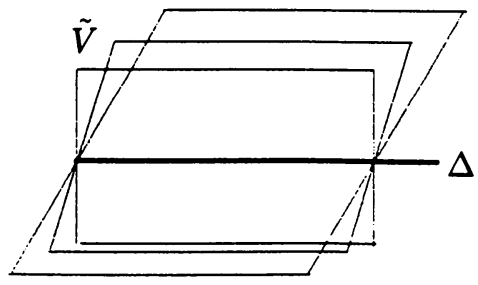

FIGURE 2

We see from the above that, while $\tilde{V}$ may be singular, its normalization $W$ will be smooth, and for many reasons it is more convenient to work with this normalization. We can realize this normalization geometrically, as the inverse image of $\tilde{V}$ in the graph of the (rational) map from $\tilde{V}$ to the Hilbert scheme $\mathscr{H}$ of zero-dimensional subschemes of degree $\delta$ in $\mathbf{P}^{2}$ that associates to a point $C$ of $V$ its nodes: clearly this map is regular at points of $V, T N$ and $T R$, and separates the sheets of $\tilde{V}$ near $\Delta$, while over the deformation space of the cusp above, the variety

$$
\begin{gathered}
\left\{\left((a, b),(\mu, \nu): y^{2}=x^{3}+a x+b \text { is singular at }(\mu, \nu)\right\}\right. \\
=\left\{((a, b),(\mu, \nu)): \nu=0, a=-3 \mu^{2}, b=2 \mu^{3}\right\}
\end{gathered}
$$



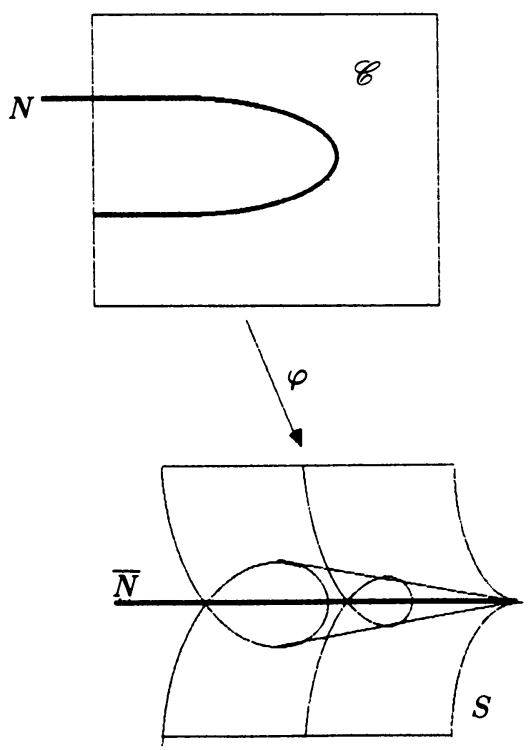

FIgURE 3. Local equation of family: $y^{2}-x^{3}+3 t^{2} x-2 t^{3}=0$; local equation of $\bar{N}: x=t, y=0$.
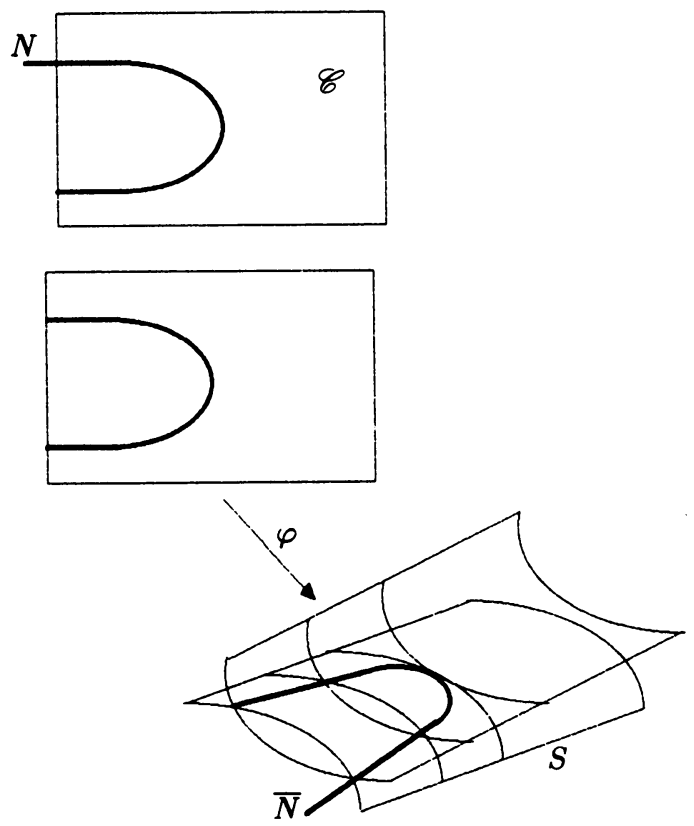

FIGURE 4. Local equation of family: $y^{2}-x^{2} y+t x^{2}-t^{2}=0$; local equation of $\bar{N}: t=\frac{1}{2} x^{2}, y=t$. 


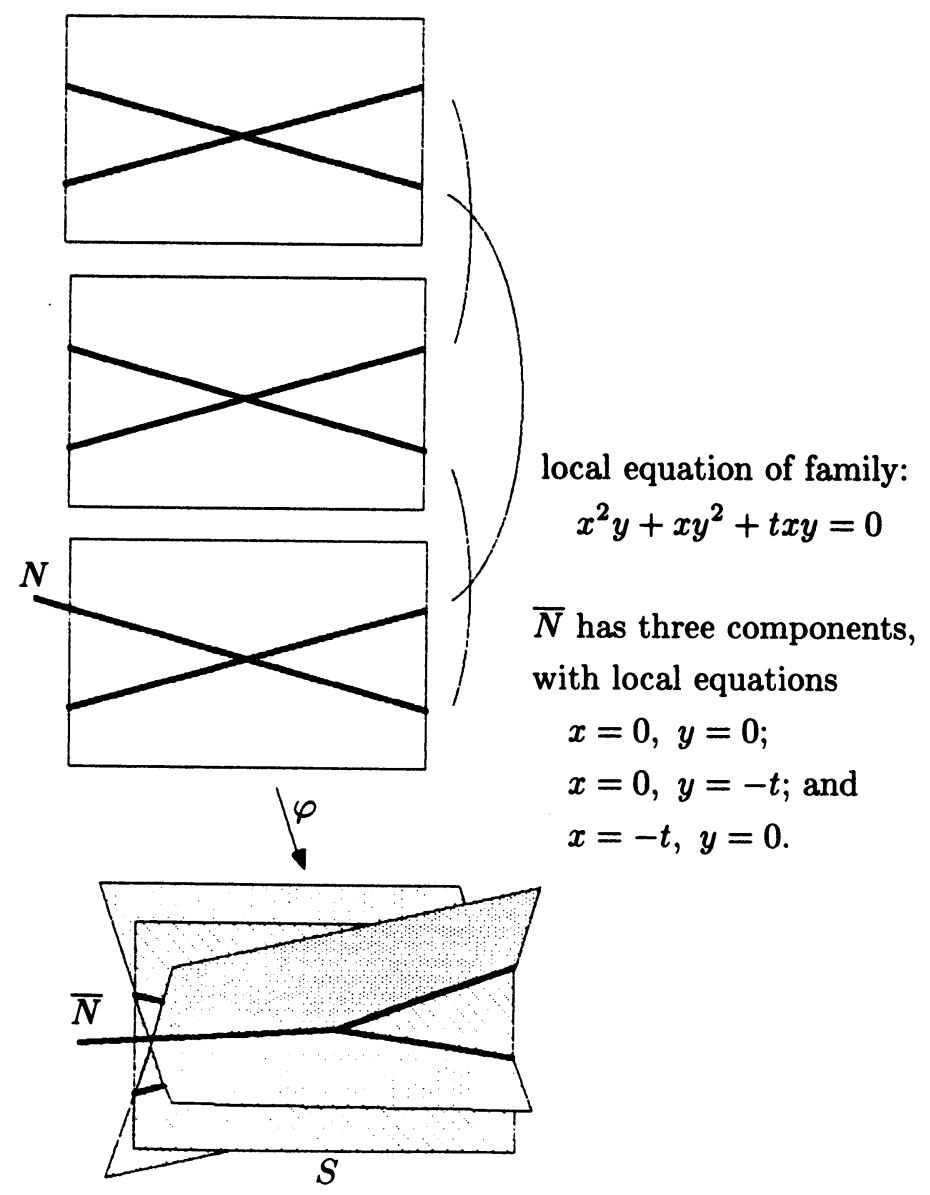

FIGURE 5. Local equation of family $x^{2} y+x y^{2}+t x y=0$. $\bar{N}$ has three components, with local equations $x=0, y=$ $0 ; x=0, y=-t ;$ and $x=-t, y=0$.

is just the normalization of the discriminant locus $4 a^{3}+27 b^{2}=0$. We can thus view the normalization $W$ as a variety of plane curves with $\delta$ assigned singularities; it is this variety that we shall deal with almost exclusively in the sequel, and that we shall call the Severi variety. We will denote the normalization map $W \rightarrow \tilde{V} \rightarrow \mathbf{P}^{N}$ by $\rho$.

REMARK. We will use the term Severi variety for different varieties-in effect, all partial compactifications of the locus $V$ of irreducible nodal curves of degree $d$ and genus $g$. (In view of the fact that we consider the variety $W$ merely a temporary expedient, enabling us to do work on $V$ in the absence of a good compactification, we do not want to appropriate the term exclusively for it.) Where the term Severi variety is used without further specification in this paper, however, we mean $W$.

(b) Local description of the family of curves over $W$. Let $\mathscr{S} \subset \mathbf{P}^{N} \times \mathbf{P}^{2}$ be the universal curve, that is, the variety whose fiber over each point of $\mathbf{P}^{N}$ is the corresponding plane curve; and let $S \subset W \times \mathbf{P}^{2}$ be the pullback via $\rho$ of this 
universal curve. From the explicit description of the deformation spaces of a cusp, tacnode and triple point, it is easy to see what $S$ looks like near each of the loci $C U, T N, T R$ and $\Delta$. In particular, we see that there is a variety $\mathscr{C} \rightarrow W$ whose fiber over each point $w \in W$ is the normalization of the corresponding plane curve $C_{w}$ at the assigned singularities; indeed, $\mathscr{C}$ will just be the normalization of the total space $S$. Explicitly, near a point of $C U$ the varieties $\mathscr{C}$ and $S$ will look like Figure 3; while near points of $T N$ and $T R$ they will be as pictured in Figure 4 and Figure 5 respectively. In each of the three figures we have labeled the locus $\bar{N}$ of assigned nodes, and the inverse image $N$ of this locus in $\mathscr{C}$. Also, note that these pictures apply not only to the Severi variety itself, but to any family $Z \subset W$ of plane curves with assigned singularities.

We now list the objects and maps we have introduced, and that we will be dealing with. We have the diagram

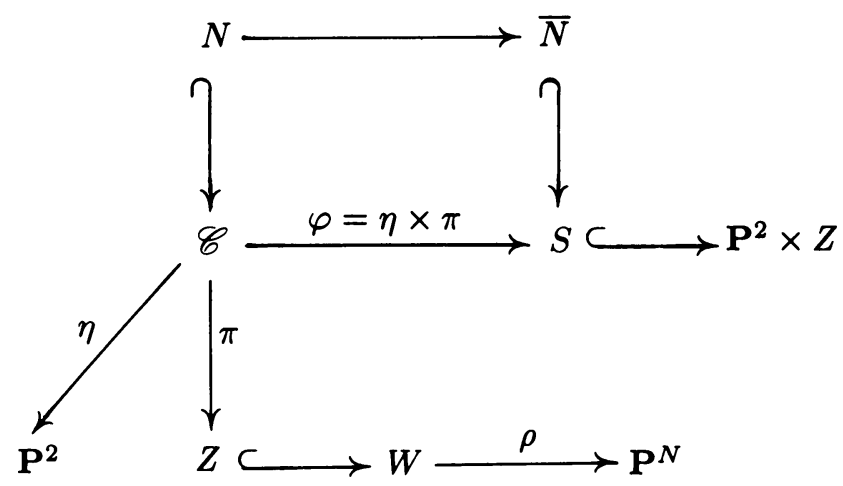

with objects and map:

the space $\mathbf{P}^{N}$ of plane curves of degree $d$;

the Severi variety $\rho: W \rightarrow \mathbf{P}^{N}$;

an arbitrary locally closed subvariety $Z \subset W$;

the family $\pi: \mathscr{C} \rightarrow Z$ of curves of arithmetic genus $g$, pulled back from the universal such family over $W$;

the map $\eta: \mathscr{C} \rightarrow \mathrm{P}^{2}$ sending each fiber $C_{z}=\pi^{-1}(z)$ of $\pi$ to the corresponding plane curve $\rho(z) \in \mathbf{P}^{N}$;

the family of plane curves $S \subset \mathbf{P}^{2} \times Z$ associated to $\rho: Z \rightarrow \mathbf{P}^{N}$; equivalently, the image of $\mathscr{C}$ in $\mathbf{P}^{2} \times Z$ via the map $\rho=\eta \times \pi$;

the locus $\bar{N} \subset S$ of assigned nodes of the plane curves $\rho(z)$ (again, if $Z$ is a smooth curve transverse to $\Delta$, this will be just the singular locus of $S$ ); and

the inverse image $N=\varphi^{-1}(\bar{N}) \subset \mathscr{C}$.

With all this said, what would we like to do with the Severi variety? Our first goal, given that we have compactified the locus of nodal curves in codimension 1 , will be to describe the divisors and line bundles on $W$. To begin with, there are many divisors on $W$ characterized as the loci in $W$ of plane curves with a given geometric property; we will call such divisors extrinsic divisors, and will discuss them in the following section. There are also a number of divisor classes that arise just from the abstract family of curves $\mathscr{C} \rightarrow W$ and the line bundle $\mathscr{L}=\eta^{*} \mathscr{O}_{\mathbf{P}^{2}}(1)$; 
we call these the intrinsic divisor classes and describe them in (d) below. Finally, we may ask what relations exist between these two classes of divisors; these relations, together with some further questions and conjectures, are stated in the final section of this chapter and proved in the remaining chapters of the paper.

(c) Extrinsic divisors. As indicated, we consider first the divisors in $W$ defined by geometric properties of the corresponding plane curves, or their position relative to other plane figures. The most obvious are those corresponding to curves whose singularities consist of other than $\delta$ nodes: the boundary components $C U, T N$, $T R$ and the $\Delta_{i, j}$. Other divisors characterized by geometric properties of the plane curves themselves are as follows.

The divisor $H F$ of curves with a hyperflex - that is, the closure of the locus of curves having contact of order four or more with their tangent line at a smooth point.

The divisor $F N$ of curves with a flecnode - that is, a node such that the tangent line to one of the branches has contact of order three or more with that branch.

The divisor $F B$ of curves with a flex bitangent - that is, a bitangent line having contact of order three or more with the curve at one of its points of tangency.

The divisor NT of curves with a nodal tangent - a line tangent to a branch of the curve at a node and tangent again elsewhere.

The divisor $B T$ of curves with a binodal tangent--i.e., such that the line joining two nodes is tangent to a branch at one of them.

The divisor $T T$ of curves with a tritangent line.

The divisor $C C$ of curves with three collinear nodes.

Note that all of the above are components of the locus of curves having a total intersection number $m+3$ with a line at $m$ points. The other components of this locus, apart from the divisors $T N$ and $T R$, are the families of curves such that the line joining two nodes is tangent to the curve somewhere else; such that a bitangent line to the curve passes through a node; and such that a flex line to the curve passes through a node. We could similarly define loci of curves having a total intersection $m+6$ with a conic at $m$ points (starting with the locus of curves with septatic points); the point here is just that there are a lot of these divisors.

Other loci in $W$ can be characterized by the position of the corresponding curves relative to a fixed point $p$ or fixed line $L$, as for example:

the divisor $C P$ of curves containing the point $p$, the divisor $N L$ of curves with a node located somewhere on $L$, the divisor $T L$ of curves tangent to $L$, the divisor $F P$ of curves with a flex line passing through $p$, the divisor $F L$ of curves with a flex located somewhere on $L$, the divisor $B P$ of curves with a bitangent line through $p$, the divisor $N P$ of curves such that the tangent line to a branch of a node passes through $p$,

and so on. Again, the main point is that there are many such divisors; and it is far from apparent what relations exist among them modulo linear equivalence.

We should also introduce here three divisors on $\mathscr{C}$ as well: we have already introduced the divisor $N$ of points lying over assigned nodes of the corresponding 
plane curves; in addition we have

the divisor $T$ of points whose tangent lines pass through the point $p$; and the divisor $F$ of points lying over flexes.

(d) Intrinsically defined divisor classes. There is another approach to the Picard group of $W$ that offers at least the hope of introducing some order into the profusion of divisors above. This is to focus on the intrinsic divisor classes - that is, divisor classes defined in terms of the varieties and maps in the diagram above.

To make an analogy, to describe the Picard group of the moduli space $\mathscr{M}_{g}$ of curves of genus $g$, we consider the universal curve $\pi: \mathscr{C}_{g} \rightarrow \mathscr{M}_{g}$ (of course, the universal curve exists only over the locus $\mathscr{M}_{g}^{0}$ of curves without automorphisms; but since the complement of this locus has codimension $g-2$, this will not affect the Picard group of $\mathscr{M}_{g}$ for $g>3$ ). On $\mathscr{C}_{g}$ we have a naturally defined divisor class, which is just the first Chern class $\omega$ of the relative cotangent bundle. We can then define a class $\kappa_{1}$ on $\mathscr{M}_{g}$ by taking the Gysin image $\pi_{*}\left(\omega^{2}\right)$ of the square of this class; and indeed it turns out that $\kappa_{1}$ generates $\operatorname{Pic}\left(\mathscr{M}_{g}\right)$, at least over $\mathbf{Q}$. (Further, it is a legitimate question whether more generally the Gysin images $\kappa_{1}=\pi_{*}\left(\omega^{i+1}\right)$ generate the cohomology/Chow ring of $\mathscr{M}_{g}$ in degrees up to roughly $g / 3 ; \mathrm{cf}$. [Harris, AMS notes]). If we now want the Picard group of the stable compactification $\overline{\mathscr{M}}_{g}$ of $\mathscr{M}_{g}$ we have only to throw in the boundary components $\Delta_{\alpha}$.

In our present circumstances, $W$ is a moduli space not for abstract curves, but for curves with a projective embedding. Correspondingly, we have over $W$ a universal family $\mathscr{C}$ of curves of genus $g$, and not one but two divisor classes: the first Chern class $\omega$ of the relative dualizing sheaf of $\mathscr{C}$ over $W$, and the pullback $D=\eta^{*}\left(c_{1}\left(\mathscr{O}_{\mathbf{p}^{2}}(1)\right)\right)$. A natural thing to do to define divisor classes on $W$ is to take all three pairwise products of these two classes and push them forward; specifically, we define classes in $\mathrm{Pic}(W)$

$$
A=\pi_{*}\left(D^{2}\right), \quad B=\pi_{*}(D \cdot \omega) \quad \text { and } \quad C=\pi_{*}\left(\omega^{2}\right)
$$

Of course, we have to include loci in $W$ over which the fibers of $\mathscr{C}$ are singular. Here again we have to consider not only the abstract fiber $C$ but the degrees of the divisor $D$ on the components of $C$ : for each $i$ such that $0 \leq i \leq g / 2$ and $(j-1)(j-2) / 2 \geq i \geq(j-1)(j-2) / 2-\delta$, we define $\Delta_{i, j}$ to be the locus of points $w \in W$ such that the fiber $\pi^{-1}(w)$ is given as the union of components $C_{0}$ and $C_{1}$ of genera $i$ and $g-i$ meeting at one point, and such that the degree of $D$ on $C_{0}$ is $j$ (obviously, if $i=g / 2$ the divisors $\Delta_{i, j}$ and $\Delta_{i, d-j}$ are the same). We also define $\Delta_{0}$ to be the locus in $W$ over which the fibers of $\pi$ are irreducible nodal curves (by [Harris] the divisors $\Delta_{i, j}$ and $\Delta_{0}$ are all irreducible). As before, we will denote by $\Delta$ the sum of all these divisors.

(e) Relations and conjectures. What relations exist among the various divisor classes introduced so far? Our principal result is simply that all the extrinsic divisors defined above are linearly equivalent to rational linear combinations of the divisor classes $A, B, C$ and the boundary components. We will in fact prove, in the following chapter, that a very broad class of such divisors are so expressible, and in Chapters 2, 3 and 4 we determine the classes of several of the divisors listed above. We state our results here. First, we have formulas for the classes of the boundary 
components $C U, T N$, and $T R$ :

$$
\begin{aligned}
& C U \sim 3 A+3 B+C-\Delta . \\
& T N \sim(3(d-3)+2 g-2) A+(d-9) B-\frac{5}{2} C+\frac{3}{2} \Delta . \\
& T R \sim\left(\frac{d^{2}-6 d+8}{2}-g+1\right) A-\frac{d-6}{2} B+\frac{2}{3} C-\frac{1}{3} \Delta .
\end{aligned}
$$

We observe one immediate consequence of these formulas: since the linear combination

$$
(7 d-18) \cdot C U+(6 d-6) \cdot T N+(12 d-198) \cdot T R+(7 d-80) \cdot \Delta
$$

has positive coefficients for $d \geq 17$ and is by (1.1)-(1.3) linearly equivalent to a positive multiple of the divisor class $A$, which is ample on the closure $\bar{V}$ of the variety $V$ of nodal curves, we deduce that $V$ is affine whenever $d \geq 17$. Other positive linear combinations of the boundary components likewise yield ample divisors on compactifications of $V$ when $d<17$, so that we may deduce the

COROLLARY. The variety $V$ of nodal curves of given degree and genus is affine.

It follows in particular that $V$ contains no complete curves, i.e., that there does not exist a complete family of nodal plane curves with constant geometric genus.

As for the classes of the other divisors described above, one is obvious: the locus $C P$ of curves containing a point $p \in \mathbf{P}^{2}$ is just the image $\pi\left(\eta^{-1}(p)\right)$; its class is thus $\pi_{*}\left(D^{2}\right)=A$. As for the rest, we have:

$$
N L \sim(2 d-3) A / 2-B / 2 \text {. }
$$

$$
T L \sim A+B \text {. }
$$

$$
F P \sim 3 A+6 B+2 C-\Delta .
$$

$$
\begin{aligned}
& F L \sim 3 A+3 B-\Delta_{0,1} . \\
& F N \sim(6 d+6 g-21) A+(3 d-18) B-5 C+2 \Delta-(d-2) \Delta_{0,1} .
\end{aligned}
$$$$
N P \sim(4 d+2 g-11) A+(d-8) B-2 C+\Delta .
$$$$
H F \sim 6 A+18 B+11 C-5 \Delta+4 \Delta_{0,1} \text {. }
$$

$$
\begin{aligned}
& N \sim(d-3) D-\omega+\pi^{*} A . \\
& T \sim 2 D+\omega .
\end{aligned}
$$

$$
F \sim 3 D+3 \omega-\Delta_{0,1} \text {. }
$$

$$
B R_{N} \sim\left(3 d^{2}-12 d+9-(2 g-2)\right) A-(d-3) B .
$$

$B R_{T} \sim 4 A+6 B+2 C$.

(Here $B R$ stands for the branch divisor of the subscripted divisor in $\mathscr{C}$, viewed as a branched covering of $W$.)

Emboldened by these examples, by the techniques of the following chapter, and by the analogy with the moduli of abstract curves, we (some of us, anyway) may make the

CONJECTURE. The Picard group of the Severi variety $W$ is generated over $\mathbf{Q}$, by the classes $A, B$, and $C$ and the classes of the boundary components $\Delta_{0}$ and $\Delta_{i, j}$. 
We do not see, at present, any way to prove this; the basis for the conjecture is simply the fact that every divisor that has been described in the Severi variety can be seen to be in the span of the intrinsic divisor classes. Observe that since the coefficients of the three classes $A, B$, and $C$ in the formulas (1.1)-(1.3) for the classes of $C U, T N$ and $T R$ are independent, $A, B$ and $C$ are themselves rational linear combinations of $C U, T N, T R$ and the boundary components $\Delta_{0}$ and $\Delta_{i, j}$. The restrictions of $A, B$ and $C$ to the variety $V$ of nodal curves are thus torsion classes, and the conjecture above is equivalent to the

\section{CONJECTURE'. The Picard group of the variety $V$ of nodal cures is torsion.}

In a future paper, the authors will show that, with the exception of the cases $g=$ 0 or 1 and $\delta=0,1$ or 2 , the divisor classes $A, B, C$ and $\Delta$ are indeed independent.

Note that there is one divisor class on the Severi variety that has not yet been mentioned: the canonical class $K_{W}$. In the final chapter of this paper, we determine this class; we find that

$$
K_{W}=-\frac{3}{2} A+\frac{3}{2} B+\frac{11}{12} C-\frac{13}{12} \Delta .
$$

We do not know for what values of $d$ and $g$ this class is actually effective.

Finally, we note that while we are dealing here with a parameter space for curves in $\mathbf{P}^{2}$, for some purposes one might want to take the quotient of $W$ by the action of $P G L_{3}$ and look at the moduli space for triples $(C, \mathscr{L}, V)$, where $C$ is a curve, $\mathscr{L}$ a line bundle on $C$, and $V \subset H^{0}(C, \mathscr{L})$ a linear system mapping $C$ birationally onto a curve of the appropriate type. Such a quotient exists, at least when the degree $d \geq 5$, since all the curves in $W$ will be stable, and the results of this paper, suitably rephrased, apply in this context. Specifically, for any family of triples $\left\{\left(C_{\lambda}, \mathscr{L}_{\lambda}, V_{\lambda}\right)\right\}_{\lambda \in Z}$ - that is, a family $\pi: \mathscr{C} \rightarrow Z$ of curves, with a line bundle $\mathscr{L}$ on $\mathscr{C}$ defined up to twists by pullbacks of line bundles from $Z$ and a subbundle $\mathscr{V} \subset \pi_{*} \mathscr{L}$ of rank 3 -we already have a divisor class $\omega=c_{1}\left(\omega_{\mathscr{C} / Z}\right)$ on $\mathscr{C}$, and we can define a (rational) divisor class $D=c_{1}(\mathscr{L})$ by normalizing $\mathscr{L}$ so that $c_{1}(\mathscr{V})=0$-that is, by setting $D=c_{1}(\mathscr{L})-\pi^{*} c_{1}(\mathscr{V}) / 3$. In this way, we can define rational classes $A, B$, and $C$ on $Z$. Of the extrinsic divisors, the ones invariant under $P G L_{3}$ of course define divisors on the quotient; the others can be defined in terms of their relations with $A, B$, and $C$ (for example, the class of the divisor $C P$ of curves passing through a point can simply be defined to be the divisor class $A=\pi_{*}\left(D^{2}\right)$ ). With this understood, all the formulas of this paper continue to hold.

ACKNOWLEDGMents. We would very much like to thank Bill Fulton, who first introduced us to these questions.

2. Relations among divisor classes via Porteous' formula. In this and the following two chapters we will show how the extrinsic divisors $C U, T N, T R$ and others may be expressed in terms of the intrinsic classes $A, B, C$ and $\Delta$. These problems naturally break up into two parts: first, if we work only over the complement of $\Delta$ in $W$, they may all be set up readily as applications of Porteous' and Porteous-type formulas; this is what we will do in this section. This will prove that the extrinsic divisors are linear combinations of the intrinsic ones, and tell us the coefficients of $A, B$, and $C$; to find the coefficients of the components of $\Delta$ will require the more ad-hoc analysis carried out in $\S \S 3$ and 4 . 
We will keep the notation introduced in the last chapter. Recall in particular that we denote by $D$ the class of the bundle $\mathscr{O}(1)$ on $\mathbf{P}^{2}$, and also its pullback to $\mathbf{P}^{2} \times Z$, $S$ and $\mathscr{C}$; we will also denote by $\mathscr{L}$ the associated line bundle $\mathscr{O}(D)=\eta^{*} \mathscr{O}_{\mathbf{P}^{2}}(1)$ and by $\mathscr{L}_{C}$ its restriction to a fiber $C$ of $\pi$. In addition, we will denote by $\omega$ the first Chern class of the relative dualizing sheaf $\omega_{\mathscr{C} / Z}$.

(a) Cusps. We start with the divisor class $C U$ of curves with a cusp. As we indicated, the computations of the classes $C U, T N$ and $T P$ will be made first over the complement of $\Delta$ in $W$; for the remainder of this section, then, we will assume $Z \subset W-\Delta$.

We can characterize points of $\mathscr{C}$ that become cusps of the corresponding curves in the plane as follows: let $p \in \mathscr{C}$ be a point, and let $C$ be the fiber of $\pi$ through $p$. Then the image $\eta(p)$ will be a cusp of the curve $\eta(C)$ if the divisor $2 \cdot p$ fails to impose independent conditions on the linear system $\eta^{*} H^{0}\left(\mathbf{P}^{2}, \mathscr{O}(1)\right) \subset H^{0}(C, \mathscr{L})$; that is, if there are two independent elements of $\eta^{*} H^{0}\left(\mathbf{P}^{2}, \mathscr{O}(1)\right)$ whose restriction of $C$ vanishes to order 2 at $p$.

To determine the class of the locus of points where this happens, we set up a bundle map. First, we let $\mathscr{E}$ be the trivial vector bundle of rank 3 on $\mathscr{C}$ whose fiber at every point is just the vector space $H^{0}\left(\mathbf{P}^{2}, \mathscr{O}(1)\right)$. Secondly, we define a vector bundle $\mathscr{F}_{(2)}$ of rank 2 whose fiber at a point $p$ of $\mathscr{C}$ is the space of sections of the sheaf $\mathscr{L}_{C} / \mathscr{L}_{C}(-2 p)$, that is, the space of local sections of the restriction of $\mathscr{L}$ to $C$, modulo those vanishing to order 2 at $p$. Precisely, we can define $\mathscr{F}_{(2)}$ as the direct image

$$
\mathscr{F}_{(2)}=\pi_{1 *}\left(\pi_{2}^{*} \mathscr{L} \otimes \mathscr{O} / \mathscr{O}(-2 \Delta)\right)
$$

where $\pi_{1}$ and $\pi_{2}$ are the projections from $\mathscr{C}_{2}=\mathscr{C} \times{ }_{z} \mathscr{C}$ to $\mathscr{C}$ and $\Delta \subset \mathscr{C}_{2}$ is the diagonal. We then have a natural map $\varphi$ from $\mathscr{E}$ to $\mathscr{F}_{(2)}$, given naively at $p$ by taking a section $\sigma \in H^{0}\left(\mathbf{P}^{2}, \mathscr{O}(1)\right)$, pulling it back by $\eta$, restricting it to $C$ and evaluating it at $p$; or more precisely, given the inclusion of $E$ in $\pi^{*}\left(\pi_{*} \mathscr{L}\right)$, as the direct image of the quotient $\operatorname{map} \pi_{2}^{*} \mathscr{L} \rightarrow \pi_{2}^{*} \mathscr{L} \otimes \mathscr{O} / \mathscr{O}(-2 \Delta)$. In any event, the locus in $\mathscr{C}$ of points mapping via $\eta$ to cusps of their fiber is the locus where $\varphi$ fails to have rank 2, and the class of this locus is simply the second Segre class of the bundle $\mathscr{F}_{(2)}$ where the Segre class of a bundle is the inverse of the Chern class (see for example [Fulton, book]). We have thus

$$
C U=\left[\pi_{*}\left(c\left(\mathscr{F}_{(2)}\right)^{-1}\right)\right]_{1}
$$

and it remains to calculate this quantity.

Now, the Chern class of $\mathscr{F}_{(2)}$ can be calculated (as it is, for example, in [Harris, AMS notes]) by observing that if $\mathscr{F}^{\prime} \subset \mathscr{F}_{(2)}$ is the subbundle whose fiber at every point $p$ is the space of sections of $\mathscr{L}_{C}(-p) / \mathscr{L}_{C}(-2 p)$, then we have isomorphisms $\mathscr{F}_{(2)} / \mathscr{F}^{\prime} \cong \mathscr{L}$ and $\mathscr{F}^{\prime} \cong \mathscr{L} \otimes \omega ;$ thus

$$
c\left(\mathscr{F}_{(2)}\right)=(1+D) \cdot(1+D+\omega)
$$

and

$$
\begin{aligned}
c\left(\mathscr{F}_{(2)}\right)^{-1} & =\left(1-D+D^{2}+\cdots\right) \cdot\left(1-(D+\omega)+(D+\omega)^{2}+\cdots\right) \\
& =1-(2 D+\omega)+\left(3 D^{2}+3 D \cdot \omega+\omega^{2}\right)+\cdots .
\end{aligned}
$$

We have thus

$$
C U=\pi_{*}\left(3 D^{2}+3 D \cdot \omega+\omega^{2}\right)=3 A+3 B+C,
$$

verifying (1.1) modulo the boundary components $\Delta$. 
(b) Tacnodes. Next, we look at tacnodes. Here the idea will be similar, but a few technical complications arise. To begin with, we ask when a pair of points $p$ and $q$ on the same fiber $C$ of $\pi$ will map to a tacnode of the image $\eta(C)$. In order for this to happen, of course, they must map to the same point of the planemeaning that the divisor $p+q$ must fail to impose two conditions on the linear series $\eta^{*} H^{0}\left(\mathbf{P}^{2}, \mathscr{O}(1)\right)$ restricted to $C$. Their tangent lines must then coincide; this means that there is a divisor in the linear series $\eta^{*} H^{0}\left(\mathbf{P}^{2}, \mathscr{O}(1)\right)$ containing $p$ and $q$ both doubly, or equivalently that the divisor $2 p+2 q$ imposes only two conditions on the linear series $\eta^{*} H^{0}\left(\mathbf{P}^{2}, \mathscr{O}(1)\right)$. To describe the locus of pairs $(p, q)$ for which this happens, we introduce a pair of bundles: $\mathscr{F}_{(1,1)}$ will be the bundle over $\mathscr{C}_{2}$ whose fiber at a point $(p, q)$ is the space of sections of $\mathscr{L}_{C} / \mathscr{L}_{C}(-p-q)$, and $\mathscr{F}(2,2)$ the bundle with fiber $\Gamma\left(\mathscr{L}_{C} / \mathscr{L}_{C}(-2 p-2 q)\right)$ over $(p, q)$. Precisely, if $\mathscr{L}_{3}$ denotes the triple fiber product of $\mathscr{C}$ with itself over $Z$, then

$$
\mathscr{F}_{(1,1)}=\pi_{1,2 .}\left(\pi_{3}^{*} \mathscr{L} \otimes \mathscr{O} \mid \mathscr{O}\left(-\Delta_{13}-\Delta_{23}\right)\right)
$$

where $\pi_{i_{1} i_{2}, \ldots}$ denotes projection on the product of the $i_{\alpha}$ th factors, and $\Delta_{i j}$ denotes the diagonal where the $i$ th and $j$ th factors are equal; and similarly

$$
\mathscr{F}_{(2,2)}=\pi_{1,2 .}\left(\pi_{3}^{*} \mathscr{L} \otimes \mathscr{O} / \mathscr{O}\left(-2 \Delta_{13}-2 \Delta_{23}\right)\right) \text {. }
$$

Clearly, $\mathscr{F}_{(1,1)}$ is a quotient of $\mathscr{F}_{(2,2)}$; and we have by evaluation a diagram of bundle maps

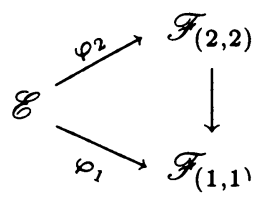

and, by what was said above, the locus $\Omega$ in $\mathscr{C}_{2}$ of pairs $(p, q)$ mapping to a tacnode is the locus where $\varphi_{1}$ has rank 1 and $\varphi_{2}$ rank 2. The class of this locus is then given, by a generalization of Porteous' formula: taking transposes, we have maps

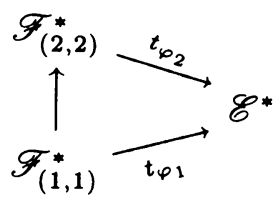

and by [Fulton, book, Theorem 14.3] the class of $\Omega$ is given by the determinant

$$
\left|\begin{array}{ll}
c_{2}\left(\mathscr{E}^{*}-\mathscr{F}_{(1,1)}^{*}\right) & c_{3}\left(\mathscr{E}^{*}-\mathscr{F}_{(1,1)}^{*}\right) \\
c_{0}\left(\mathscr{E}^{*}-\mathscr{F}_{(2,2)}^{*}\right) & c_{1}\left(\mathscr{E}^{*}-\mathscr{F}_{(2,2)}^{*}\right)
\end{array}\right|=s_{2}\left(\mathscr{F}_{(1,1)}^{*}\right) \cdot s_{1}\left(\mathscr{F}_{(2,2)}^{*}\right)-s_{3}\left(\mathscr{F}_{(1,1)}^{*}\right)
$$

where $s(\mathscr{F})$ is the Segre class $c(\mathscr{F})^{-1}$

$$
\begin{aligned}
= & \left(c_{1}\left(\mathscr{F}_{(1,1)}\right)^{2}-c_{2}\left(\mathscr{F}_{(1,1)}\right)\right) \cdot c_{1}\left(\mathscr{F}_{(2,2)}\right) \\
& -c_{1}\left(\mathscr{F}_{(1,1}\right)^{3}+2 c_{1}\left(\mathscr{F}_{(1,1)}\right) c_{2}\left(\mathscr{F}_{(1,1)}\right)
\end{aligned}
$$

and the divisor $T N$ will then be the pushforward, via the map $\pi: \mathscr{C}_{2} \rightarrow Z$, of this class (by abuse of language, we will use $\pi$ to denote the projection from $\mathscr{C}_{k}$ to $Z$ for any $k$ ). 
The next step is to determine the Chern classes of the sheaves $\mathscr{F}$. Here a naive approach like last time doesn't work; instead we have to use the definition of the sheaves $\mathscr{F}$ and apply the Grothendieck-Riemann-Roch formula as in [ACGH]. Consider, for example, the description (2.1) of the sheaf $\mathscr{F}_{(1,1)}$. Since the sheaf $\pi_{3}^{*} \mathscr{L} \otimes \mathscr{O} / \mathscr{O}\left(-\Delta_{13}-\Delta_{23}\right)$ is supported on a subvariety of $\mathscr{C}$ finite over $\mathscr{C}_{2}$, it will have no higher direct images under the map $\pi_{1,2}$; thus by the GrothendieckRiemann-Roch the Chern character of its direct image under $\pi_{1,2}$ will be given by

$$
\begin{aligned}
\operatorname{ch}\left(\pi_{1,2 *}\left(\pi_{3}^{*} \mathscr{L} \otimes \mathscr{O} / \mathscr{O}\left(-\Delta_{13}-\Delta_{23}\right)\right)\right) \\
\quad=\pi_{1,2 *}\left(\operatorname{Td}\left(\mathscr{C}_{3} / \mathscr{C}_{2}\right) \cdot \operatorname{ch}\left(\pi_{3}^{*} \mathscr{L} \otimes \mathscr{O} / \mathscr{O}\left(-\Delta_{13}-\Delta_{23}\right)\right)\right) \\
\quad=\pi_{1,2 *}\left(\left(1-\omega_{3} / 2+\omega_{3}^{2} / 12\right) \cdot\left(e^{D_{3}} \cdot\left(1-e^{-\Delta_{13}-\Delta_{23}}\right)\right)\right)
\end{aligned}
$$

where $\omega_{i}$ and $D_{i}$ denote the pullback to $\mathscr{C}_{3}$ via $\pi_{i}$ of the classes $\omega$ and $D$ respectively

$$
=\pi_{1,2 .}\left(\left(1-\omega_{3} / 2+\omega_{3}^{2} / 12\right) \cdot\left(1+D_{3}+D_{3}^{2} / 2\right) \cdot\left(\Delta-\Delta^{2} / 2+\Delta^{3} / 6+\Delta^{4} / 24\right)\right)
$$

(where $\Delta=\Delta_{13}+\Delta_{23}$ ) modulo classes supported over loci of codimension greater than one in $Z$. We can evaluate this expression by applying relations in the Chow ring of $\mathscr{C}_{3}$ : we have

$D_{i}^{3}=\omega_{i}^{3}=\Delta_{i j}^{4}=0$ for all $i \neq j$, again modulo classes supported on loci of codimension greater than one in $Z$;

$D_{i} \cdot \Delta_{i j}=D_{j} \cdot \Delta_{i j}$, and $\omega_{i} \cdot \Delta_{i j}=\omega_{j} \cdot \Delta_{i j} \quad$ for all $i, j$;

$\Delta_{i j} \cdot \Delta_{j k}=\Delta_{i j} \cdot \Delta_{i k}$ for all $i \neq j \neq k \neq i$;

$\Delta_{i j}^{2}=-\omega_{i} \cdot \Delta_{i j} ;$ and

$\pi_{1,2 .}\left(D_{i} \cdot \Delta_{i 3}\right)=D_{i}$ and $\pi_{1,2 .}\left(\omega_{i} \cdot \Delta_{i 3}\right)=\omega_{i}$ for $i=1,2$; and using these relations allow us to evaluate the last expression. Explicitly, we have

$$
\begin{aligned}
& \operatorname{ch}_{1}\left(\mathscr{F}_{(1,1)}\right)=\pi_{1,2 .}\left(-\omega_{3} \cdot\left(\Delta_{13}+\Delta_{23}\right) / 2\right. \\
& \left.+D_{3} \cdot\left(\Delta_{13}+\Delta_{23}\right)-\left(\Delta_{13}+\Delta_{23}\right)^{2} / 2\right) \\
& =\pi_{1,2 .}\left(-\omega_{3} \cdot\left(\Delta_{13}+\Delta_{23}\right) / 2+D_{3} \cdot\left(\Delta_{13}+\Delta_{23}\right)\right. \\
& \left.+\omega_{1} \cdot \Delta_{13} / 2-\Delta_{12} \cdot \Delta_{13}+\omega_{2} \cdot \Delta_{23} / 2\right) \\
& =-\omega_{1} / 2-\omega_{2} / 2+D_{1}+D_{2}+\omega_{1} / 2+\omega_{2} / 2-\Delta_{12} \\
& =D_{1}+D_{2}-\Delta_{12} \text {, } \\
& \operatorname{ch}_{2}\left(\mathscr{F}_{(1,1)}\right)=\pi_{1,2 .}\left(\left(\Delta_{13}+\Delta_{23}\right) \cdot\left(\omega_{3}^{2} / 12-\omega_{3} \cdot D_{3} / 2+D_{3}^{2} / 2\right)\right. \\
& \left.-\left(\Delta_{13}+\Delta_{23}\right)^{2} \cdot\left(-\omega_{3} / 2+D_{3}\right) / 2+\left(\Delta_{13}+\Delta_{23}\right)^{3} / 6\right) \\
& =\pi_{1,2 .}\left(\left(\Delta_{13}+\Delta_{23}\right) \cdot\left(\omega_{3}^{2} / 12-\omega_{3} \cdot D_{3} / 2+D_{3}^{2} / 2\right)\right. \\
& +\omega_{1} \cdot\left(D_{1}-\omega_{1} / 2\right) \cdot \Delta_{13} / 2+\omega_{2} \cdot\left(D_{2}-\omega_{2} / 2\right) \cdot \Delta_{23} / 2 \\
& -\left(D_{1}-\omega_{1} / 2\right) \cdot \Delta_{12} \cdot \Delta_{23}+\omega_{1}^{2} \cdot \Delta_{13} / 6+\omega_{2}^{2} \cdot \Delta_{23} / 6 \\
& \left.-\omega_{1} \cdot \Delta_{12} \cdot \Delta_{13} / 2-\omega_{2} \cdot \Delta_{12} \cdot \Delta_{23} / 2\right) \\
& =\omega_{1}^{2} / 12+\omega_{2}^{2} / 12-\omega_{1} \cdot D_{1} / 2-\omega_{2} \cdot D_{2} / 2+D_{1}^{2} / 2+D_{2}^{2} / 2 \\
& +\omega_{1} \cdot D_{1} / 2-\omega_{1}^{2} / 4+\omega_{2} \cdot D_{2} / 2-\omega_{2}^{2} / 4-D_{1} \cdot \Delta_{12}+\omega_{1} \cdot \Delta_{12} / 2 \\
& +\omega_{1}^{2} / 6+\omega_{2}^{2} / 6-\omega_{1} \cdot \Delta_{12} / 2-\omega_{2} \cdot \Delta_{12} / 2 \\
& =D_{1}^{2} / 2+D_{2}^{2} / 2-D_{1} \cdot \Delta_{12}-\omega_{1} \cdot \Delta_{12} / 2 \text {. }
\end{aligned}
$$


Of course we have $c_{1}\left(\mathscr{F}_{(1,1)}\right)=\operatorname{ch}_{1}\left(\mathscr{F}_{(1,1)}\right)=D_{1}+D_{2}-\Delta_{12} ;$ and

$$
\begin{aligned}
c_{2}\left(\mathscr{F}_{(1,1)}\right)= & \operatorname{ch}_{1}\left(\mathscr{F}_{(1,1)}\right)^{2} / 2-\operatorname{ch}_{2}\left(\mathscr{F}_{(1,1)}\right) \\
= & D_{1}^{2} / 2+D_{2}^{2} / 2+D_{1} \cdot D_{2}-\omega_{1} \cdot \Delta_{12} / 2-2 D_{1} \cdot \Delta_{12} \\
& -\left(D_{1}^{2} / 2+D_{2}^{2} / 2-D_{1} \cdot \Delta_{12}-\omega_{1} \cdot \Delta_{12} / 2\right) \\
= & D_{1} \cdot D_{2}-D_{1} \cdot \Delta_{12}
\end{aligned}
$$

The computation for the Chern classes of the bundle $\mathscr{F}_{(2,2)}$ is of course identical to that above, except for the coefficients 2 appearing in (2.1) and (2.3). In any event, by (2.2) we need only determine the first Chern class; and we find

$$
c_{1}\left(\mathscr{F}_{(2,2)}\right)=\operatorname{ch}_{1}\left(\mathscr{F}_{(2,2)}\right)=2 D_{1}+2 D_{2}+\omega_{1}+\omega_{2}-4 \Delta_{12} .
$$

Plugging this into (2.2) above, we find that the class

$$
\begin{aligned}
\Omega \sim & \left(\left(D_{1}+D_{2}-\Delta\right)^{2}-\left(D_{1} D_{2}-D \Delta\right)\right) \cdot\left(2\left(D_{1}+D_{2}\right)+\omega_{1}+\omega_{2}-4 \Delta\right) \\
& -\left(D_{1}+D_{2}-\Delta\right)^{3}+2\left(\left(D_{1}+D_{2}-\Delta\right)\left(D_{1} D_{2}-D \Delta\right),\right.
\end{aligned}
$$

where we write $\Delta$ for $\Delta_{12}$ and suppress the subscript on a $D$ or $\omega$ multiplied by $\Delta$

$$
\begin{aligned}
= & D_{1}^{3}+3 D_{1}^{2} D_{2}+3 D_{1} D_{2}^{2}+D_{2}^{3}-18 D^{2} \Delta+12 D \Delta^{2}-4 \Delta^{3}+\omega_{1} D_{1}^{2} \\
& +\omega_{2} D_{1}^{2}+\omega_{1} D_{2}^{2}+\omega_{1} D_{2}^{2}+\omega_{1} D_{1} D_{2}+\omega_{2} D_{1} D_{2}-6 \omega D \Delta+\omega \Delta^{2} .
\end{aligned}
$$

Now, $\pi_{*} D_{i}^{3}=0$, as is the direct image of any triple product all of whose terms are from the same factor of $\mathscr{C}_{2}$; and we can deal with any triple product involving two terms pulled back from one factor of $\mathscr{C}_{2}$ and one term from the other by writing, for example,

$$
\begin{aligned}
\pi_{*} D_{1} \omega_{1} D_{2} & =\pi_{*}\left(D_{2} \cdot \pi_{2 .}\left(\omega_{1} \cdot D_{1}\right)\right) \\
& =\pi_{*}\left(D_{2} \cdot \pi^{*} B\right)=d \cdot B
\end{aligned}
$$

(here, as before, we use $\pi: \mathscr{C}_{k} \rightarrow Z$ to denote the projection from $\mathscr{C}_{k}$ for any $k$ ).

We can similarly deal with any term involving a single $\Delta$ by projecting first onto one (either) factor of $\mathscr{C}_{2}$; and with terms involving $\Delta^{2}$ by writing $\Delta^{2}=-\omega \Delta$. Doing this, we find that

$$
\begin{aligned}
T N \sim & \left(\pi_{*}[\Omega]\right) / 2=3 d A+3 d A-18 A-12 B-4 C \\
& +(2 g-2) A+(2 g-2) A+d B+d B-6 B-C \\
= & ((3 d-9)+(2 g-2)) \cdot A+(d-9) \cdot B-5 \cdot C / 2,
\end{aligned}
$$

verifying (1.2) modulo the boundary components $\Delta$.

(c) Triple points. It remains to determine the class of the divisor $T R$ of curves with triple points. The approach here is exactly like the previous two: we will use Porteous' formula to determine the class in the triple product $\mathscr{C}_{3}$ of the locus of triples $(p, q, r)$ mapping to triple points, and then push forward from $\mathscr{C}_{3}$ to $Z$.

To begin with, observe that a triple of points $(p, q, r)$ in a fiber $C_{z}$ of $\mathscr{C}$ will map to the same point of the image $\eta\left(C_{z}\right)$ if the divisor $p+q+r$ imposes only one condition on the linear system $\eta^{*} H^{0}\left(\mathbf{P}^{2}, \mathscr{O}(1)\right)$. To describe the locus $\Xi \subset \mathscr{C}_{3}$ of such points, we thus want to introduce a vector bundle $\mathscr{F}_{(1,1,1)}$ of rank 3 on $\mathscr{C}_{3}$ whose fiber at a point $(p, q, r)$ will be the vector space of sections of $\mathscr{L}_{C} / \mathscr{L}_{C}(-p-q-r)$; officially, we let $\mathscr{C}_{4}$ be the fourfold fiber product of $\mathscr{C}$ with itself over $Z, \Delta_{i j}$ the 
diagonal and $\pi_{i_{1}, \ldots, i_{k}}$ the projection map on the product of the $i_{\alpha}$ th factors, and set

$$
\mathscr{F}_{(1,1,1)}=\pi_{1,2,3_{*}}\left(\pi_{4}^{*} \mathscr{L} \otimes \mathscr{O} \mid \mathscr{O}\left(-\Delta_{14}-\Delta_{24}-\Delta_{34}\right)\right) .
$$

Having defined $\mathscr{F}_{(1,1,1)}$, we have a vector bundle map $\varphi: \mathscr{E} \rightarrow \mathscr{F}_{(1,1,1)}$ defined by evaluation (officially, identifying $\mathscr{E}$ with the direct image $\pi_{1,2,3 *}\left(\pi_{4}^{*} \mathscr{L}\right), \varphi$, is just the pushforward of the quotient map $\pi_{4}^{*} \mathscr{L} \rightarrow \pi_{4}^{*} \mathscr{L} \otimes \mathscr{O} / \mathscr{O}\left(-\Delta_{14}-\Delta_{24}-\Delta_{34}\right)$ ); and the locus $\Xi$ of points in $\mathscr{C}_{3}$ mapping to triple points of their fiber is then the locus where the map $\varphi$ has rank 1. The class of $\Xi$ is then given, by the standard Porteous formula, as the determinant

$$
[\Xi]=\left|\begin{array}{ll}
c_{2}\left(\mathscr{F}_{(1,1,1)}\right) & c_{3}\left(\mathscr{F}_{(1,1,1)}\right) \\
c_{1}\left(\mathscr{F}_{(1,1,1)}\right) & c_{2}\left(\mathscr{F}_{(1,1,1)}\right)
\end{array}\right|
$$

and it remains to evaluate the Chern classes of $\mathscr{F}_{(1,1,1)}$.

We do this in the same manner as above: by applying the Grothendieck-RiemannRoch formula to the description $(2.4)$ of the bundle $\mathscr{F}_{(1,1,1)}$. We have

$$
\begin{aligned}
\operatorname{ch}\left(\mathscr{F}_{(1,1,1)}\right. & =\pi_{1,2,3_{*}}\left(\operatorname{Td}\left(\mathscr{C}_{4} / \mathscr{C}_{3}\right) \cdot \operatorname{ch}\left(\pi_{4}^{*} \mathscr{L} \otimes \mathscr{O} / \mathscr{O}\left(-\Delta_{14}-\Delta_{24}-\Delta_{34}\right)\right)\right) \\
& =\pi_{1,2,3_{*}}\left(\left(1-\omega_{4} / 2+\omega_{4}^{2} / 12\right) \cdot\left(e^{D_{4}} \cdot\left(1-e^{-\Delta_{14}-\Delta_{24}-\Delta_{34}}\right)\right)\right) .
\end{aligned}
$$

Since the expansion of this product and the evaluation of the pushforward are formally identical with the calculation made above for $\mathscr{F}_{(1,1)}$, we will spare the reader (some of) the details and simply state the outcome, which is that

$$
\begin{aligned}
\operatorname{ch}\left(\mathscr{F}_{(1,1,1)}\right)= & +\left(D_{1}+D_{2}+D_{3}-\Delta_{12}-\Delta_{13}-\Delta_{23}\right) \\
+ & \left(\left(D_{1}^{2}+D_{2}^{2}+D_{3}^{2}\right) / 2-D_{1} \Delta_{124}-D_{2} \Delta_{234}-D_{3} \Delta_{134}\right. \\
& \left.-\left(\omega_{1} \Delta_{124}+\omega_{2} \Delta_{234}-\omega_{3} \Delta_{134}\right) / 2+\Delta_{123}\right) \\
+ & \left(-\left(D_{1}^{2} \Delta_{12}+D_{2}^{2} \Delta_{23}+D_{3}^{2} \Delta_{13}\right) / 2\right. \\
& \quad-\left(\omega_{1} D_{1} \Delta_{12}+\omega_{2} D_{2} \Delta_{23}+\omega_{3} D_{3} \Delta_{13}\right) / 2 \\
& \left.\quad-\left(\omega_{1}^{2} \Delta_{12}+\omega_{2}^{2} \Delta_{23}+\omega_{3}^{2} \Delta_{13}\right) / 6+\omega \Delta_{123}+D \Delta_{123}\right)
\end{aligned}
$$

and hence

$$
\begin{aligned}
c_{1}\left(\mathscr{F}_{1,1,1)}\right)= & D_{1}+D_{2}+D_{3}-\Delta_{12}-\Delta_{13}-\Delta_{23} \\
c_{2}\left(\mathscr{F}_{1,1,1)}\right)= & D_{1} D_{2}+D_{1} D_{3}+D_{2} D_{3}-D_{1} \Delta_{12}-D_{2} \Delta_{23}-D_{3} \Delta_{13} \\
& -D_{1} \Delta_{23}-D_{2} \Delta_{13}-D_{3} \Delta_{12}+2 \Delta_{123} \\
c_{3}\left(\mathscr{F}_{(1,1,1)}\right)= & 2 D \Delta_{123}+D_{1} D_{2} D_{3}-D_{1} D_{2} \Delta_{23}-D_{2} D_{3} \Delta_{13}-D_{1} D_{3} \Delta_{12} .
\end{aligned}
$$

Thus, by (2.5),

$$
\begin{aligned}
{[\Xi]=} & \sum D_{i}^{2} D_{j} D_{k}-\sum D_{i}^{2} D_{j} \Delta_{i k}-5 \sum D_{i}^{2} D_{j} \Delta_{j k} \\
& +4 \sum D_{i} D_{j} \Delta_{i j k}+4 \sum D_{i}^{2} \Delta_{i j k}+\sum D_{i} D_{j}\left(\Delta_{i k}\right)^{2} \\
& +\sum D_{i}^{2}\left(\Delta_{j k}\right)^{2}-6 \sum D_{i} \Delta_{i j} \Delta_{i j k}+4\left(\Delta_{123}\right)^{2}
\end{aligned}
$$


where the summation in each case is over cyclic permutations of $\{1,2,3\}$. Taking the pushforward to $Z$ and dividing by 6 we arrive at

$$
\begin{aligned}
T R= & d^{2} \cdot A / 2-d \cdot A / 2-5 d \cdot A / 2+2 A \\
& +2 A-d \cdot B / 2-(2 g-2) \cdot A / 2+3 B+2 C / 3 \\
= & \left(\frac{d^{2}+6 d+8}{2}-g+1\right) A-\frac{d-6}{2} B+\frac{2}{3} C
\end{aligned}
$$

verifying (1.3), again modulo the boundary components.

(d) REMARKS. What is clear from these constructions, above and beyond the coefficients of the divisors $C U, T N$ and $T R$, is that any of the divisors described in the previous section can in this way be expressed, modulo the boundary components, as a linear combination of the divisors $A, B$ and $C$. Thus, for example, the locus of curves with tritangents will be the image of the rank 2 locus of the evaluation map from $\mathscr{E}$ to the rank 6 bundle $\mathscr{F}_{(2,2,2)}$ on $\mathscr{C}_{3}$ whose fiber at a point $(p, q, r)$ is $\Gamma\left(\mathscr{L}_{C} / \mathscr{L}_{C}(-2 p-2 q-2 r)\right)$, and so expressible by Porteous' formula; the locus of curves with flex bitangents will be the rank 2 locus of the evaluation map from $\mathscr{E}$ to the analogously defined bundle $\mathscr{F}_{(2,3)}$ on $\mathscr{C}_{2}$; and so on (other examples will be worked out in $\S 4$ ). Without carrying out the computations explicitly, it is clear that the classes of these divisors will lie in the (rational) span of $A, B, C$ and the boundary components in $\operatorname{Pic}(W)$.

As for the determination of the coefficients of $\Delta$ in the classes of these divisors, we will carry this out in the following two sections, using a variety of ad-hoc techniques. We would like to say here, however, that this approach is not really satisfactory to us: apart from the question of whether these techniques will suffice, it would seem much preferable to have a way of extending the technique employed in this section to the boundary. The obstacles to doing this are nontrivial: it is not hard to see that the bundles $\mathscr{F}$ introduced above will not extend to vector bundles on $\mathscr{C}_{k}$ over all of $W$, and while in theory they will extend to vector bundles on a suitable blowup of $\mathscr{C}_{k}$, this is difficult to carry out in practice. What is clearly called for, then, is a version of Porteous' formula that applies to (at least some class of) coherent sheaves.

3. Divisors on $\mathbf{P}^{N}$ and their restrictions. Another technique for computing the classes of divisors on the Severi variety is to consider divisors on the space $\mathbf{P}^{N}$ of all curves in the plane, determine their class (i.e., degree) in $\mathbf{P}^{N}$, and then analyze their restriction/pullback to the Severi variety to obtain relations in $\operatorname{Pic}(W)$. This is what we shall do in this chapter; the relations that we arrive at will be applied in the following chapter.

Specifically, the divisors in $\mathbf{P}^{N}$ we shall consider here are the divisor of curves of degree $d$

(i) tangent to (i.e., having a point of intersection multiplicity two or more with) a fixed line;

(ii) having a point of intersection multiplicity three or more with some line of a fixed pencil;

(iii) having a point of intersection multiplicity four or more with some line; or

(iv) having a point of intersection multiplicity three or more with a line at a point on a fixed line. 
Call these divisors $M_{2}, M_{3}, M_{4}$, and $N_{3}$ respectively (the fact that they are indeed divisors, if not already apparent, will emerge in the course of the following computation). Their degrees may all be determined by one technique; we do this now.

(a) Degrees of the divisors $M$ and $N$. To begin with, we introduce the standard incidence correspondence

$$
\Sigma=\{(p, l): p \in l\} \subset \mathbf{P}^{2} \times \mathbf{P}^{2^{*}} .
$$

We will think of $\Sigma$ as the universal line over $\mathbf{P}^{2^{*}}$. Observe that the Chow ring of $\Sigma$ is generated by the pullbacks $\eta_{i}$ of the hyperplane (line) classes $c_{1}(\mathscr{O}(1))$ of the two factors, with the relations

$$
\eta_{i}^{3}=0 ; \quad \eta_{i}^{2} \eta_{j}=1 ; \quad \text { and } \quad \eta_{1} \eta_{2}=\eta_{1}^{2}+\eta_{2}^{2} .
$$

Now, let $\mathscr{G}$ be the line bundle $\pi_{1}^{*}(\mathscr{O}(d))$ on $\Sigma$, and let $\Omega$ be the relative dualizing sheaf of $\Sigma$ over the second factor $\mathbf{P}^{2^{*}}$; observe that since $\Sigma$ is a divisor of type $(1,1)$ on $\mathbf{P}^{2} \times \mathbf{P}^{2^{*}}$, and the Chern class of the relative dualizing sheaf of $\mathbf{P}^{2} \times \mathbf{P}^{2^{*}}$ over $\mathbf{P}^{2^{*}}$ is $-3 \eta_{1}$, we have

$$
c_{1}(\Omega)=-3 \eta_{1}+\left(\eta_{1}+\eta_{2}\right)=-2 \eta_{1}+\eta_{2} .
$$

We can introduce a vector bundle $\mathscr{G}_{k}$ on $\Sigma$ whose fiber at every point $(p, l)$ is the space of sections of the sheaf $\mathscr{O}_{l}(d) / \mathscr{O}_{l}(d)(-k \cdot p)=\mathscr{G}_{\mid \tilde{l}} / \mathscr{G}_{\mid \tilde{l}}(-k \cdot(-p, l))$, that is, sections of $\mathscr{G}$ restricted to the fiber $\tilde{l}=\{(q, l): q \in l\}$ of $\Sigma$ over $l$, modulo those vanishing to order $k$ at $(p, l)$. (Officially, if $\Sigma_{2}$ is the fiber product of $\Sigma$ with itself over $\mathbf{P}^{2^{*}}, \Delta \subset \Sigma_{2}$ the diagonal, and $\eta_{1}, \eta_{2}: \Sigma_{2} \rightarrow \Sigma$ the projections, $\mathscr{G}_{k}$ will be the sheaf $\eta_{1 .}\left(\eta_{2}^{*} \mathscr{G} \otimes \mathscr{O} / \mathscr{O}(-k \Delta)\right)$. The obvious quotient maps

$$
\mathscr{G}_{k} \rightarrow \mathscr{G}_{k-1} \rightarrow \cdots \rightarrow \mathscr{G}_{2} \rightarrow \mathscr{G}_{1}=\mathscr{G}
$$

give a filtration of $\mathscr{G}_{k}$ with successive quotients $\mathscr{G}, \mathscr{G} \otimes \Omega, \ldots, \mathscr{G} \otimes \Omega^{k-1}$, so that

$$
c\left(\mathscr{G}_{k}\right)=\left(1+d \eta_{1}\right) \cdot\left(1+(d-2) \eta_{1}+\eta_{2}\right) \cdots\left(1+(d-2 k+2) \eta_{1}+(k-1) \eta_{2}\right) .
$$

Now, on the product $\mathbf{P}^{N} \times \Sigma$, we have a natural map

$$
\varphi_{k}: \pi_{1}^{*}\left(\mathscr{O}_{\mathbf{P}^{N}}(-1)\right) \rightarrow \mathscr{G}_{k},
$$

which, at a point $(f,(p, l))$ of $\mathbf{P}^{N} \times \Sigma$, simply takes a scalar multiple of the polynomial $f$, restricts it to $l$, and evaluates it at $p$. The zero locus $\Psi_{k}$ of this map is, of course, the space of triples $(f,(p, l))$ such that $f_{\mid l}$ vanishes to order $k$ at $p$. By looking at the projection on the second factor $\Sigma$, we see that $\Psi_{k}$ always has codimension exactly $k$ in $\mathbf{P}^{N} \times \Sigma$ when $k \leq d$; writing $A$ for the hyperplane class on $\mathbf{P}^{N}$ or its pullback to $\mathbf{P}^{N} \times \Sigma$, we can determine its class as

$$
\left[\Psi_{k}\right]=\pi_{1_{*}}\left(c_{k}\left(\pi_{1}^{*}\left(\mathscr{O}_{\mathbf{P}^{N}}(1)\right) \otimes \mathscr{G}_{k}\right)\right)=\pi_{1_{*}}\left(\sum A^{k-i} \cdot c_{i}\left(\mathscr{G}_{k}\right)\right) .
$$

The classes of the divisors $M_{i}$ introduced above may now be calculated readily. To start, the locus $M_{2}$ of curves tangent to a fixed line $l_{0}$ is just the image in $\mathbf{P}^{N}$ of the intersection of $\Psi_{2}$ with the inverse image of the locus $\left\{\left(p, l_{0}\right)\right\} \subset \Sigma$; since this locus has class $\eta_{2}^{2}$, we have

$$
\begin{aligned}
M_{2} & \sim \pi_{1 *}\left(\sum A^{2-i} \cdot c_{i}\left(\mathscr{G}_{2}\right) \cdot \eta_{2}^{2}\right)=\pi_{1 *}\left(c_{1}\left(\mathscr{G}_{2}\right) \cdot \eta_{2}^{2}\right) \cdot A \\
& =\pi_{1 .}\left(\left((2 d-2) \eta_{1}+\eta_{2}\right) \cdot \eta_{2}^{2}\right) \cdot A=(2 d-2) A .
\end{aligned}
$$


Of course, we did not need any fancy machinery to tell us this. We know that in the projective space $\mathbf{P}=\mathbf{P} H^{0}\left(l_{0}, \mathscr{O}(d)\right)$ of polynomials of degree $d$ in two variables the subvariety of those with multiple roots - the zero locus of the discriminant polynomial on $\mathbf{P}$-is a hypersurface of degree $2 d-2$. Since the map $\mathbf{P}^{N} \rightarrow \mathbf{P}$ given by restriction is a linear projection, it is likewise true that the subvariety of $\mathbf{P}^{N}$ of curves having a multiple point of intersection with $l_{0}$ is a hypersurface of degree $2 d-2$.

The case of $M_{3}$ is handled similarly: for any point $p_{0}$, the locus of curves having contact of order at least three with a line through $p_{0}$ will be the image in $\mathbf{P}^{N}$ of the intersection of $\Psi_{3}$ with the inverse image of the locus $\left\{(p, l) p_{0} \in l\right\}$; since this locus has class $\eta_{2}$ we have

$$
\begin{aligned}
M_{3}= & \pi_{1 *}\left(\sum A^{3-i} \cdot c_{i}\left(\mathscr{G}_{3}\right) \cdot \eta_{2}\right) \\
= & \pi_{1 *}\left(c_{2}\left(\mathscr{G}_{3}\right) \cdot \eta_{2}\right) \cdot A \\
= & \pi_{1 *}\left(\left(\left(d \eta_{1} \cdot\left((d-2) \eta_{1}+\eta_{2}\right)+d \eta_{1} \cdot\left((d-4) \eta_{1}+2 \eta_{2}\right)\right.\right.\right. \\
& \left.\left.\quad \quad \quad\left((d-2) \eta_{1}+\eta_{2}\right) \cdot\left((d-4) \eta_{1}+2 \eta_{2}\right)\right) \cdot \eta_{2}\right) \cdot A \\
= & \left(3 d^{2}-6 d\right) A
\end{aligned}
$$

In the same vein, the class of the divisor $N_{3}$ of curves with a flex point lying on a fixed line is given as

$$
\begin{aligned}
N_{3} & \sim \pi_{1 *}\left(\sum A^{3-i} \cdot c_{i}\left(\mathscr{G}_{3}\right) \cdot \eta_{1}\right) \\
= & \pi_{1 *}\left(c_{2}\left(\mathscr{G}_{3}\right) \cdot \eta_{1}\right) \cdot A \\
= & \pi_{1 *}\left(\left(\left(d \eta_{1} \cdot\left((d-2) \eta_{1}+\eta_{2}\right)+d \eta_{1} \cdot\left((d-4) \eta_{1}+2 \eta_{2}\right)\right.\right.\right. \\
& \left.\left.\quad+\left((d-2) \eta_{1}+\eta_{2}\right) \cdot\left((d-4) \eta_{1}+2 \eta_{2}\right)\right) \cdot \eta_{1}\right) \cdot A \\
= & (6 d-6) A .
\end{aligned}
$$

Finally, the divisor $M_{4}$ is simply the image in $\mathbf{P}^{N}$ of the locus $\Psi_{4}$; so that (omitting the intermediate calculations) we have

$$
M_{4} \sim \pi_{1 .}\left(c_{3}\left(\mathscr{G}_{4}\right)\right) \cdot A=\left(18 d^{2}-66 d+36\right) \cdot A .
$$

(b) Pullbacks of the divisors $M$ and $N$. We consider now what we get when we pull back the divisors introduced above to the Severi variety $W$. In each case, we will see that the support of the pullback is readily described, while the determination of the multiplicities may require some work. (In each of the cases worked out below, the existence of families of $W$ as described follows either from an ad hoc argument or from deformation theory as found e.g. in [Diaz-Harris]).

Consider first the divisor $M_{2}$ of curves having a multiple point of intersection with a fixed line $L$. Let $C \in W$ be in the pullback of $M_{2}$, and let $p$ be a multiple point of intersection of $C$ with $L$. Then either $p$ is a smooth point of $C$-so that $C$ lies in the divisor $T L \subset W$-or $p$ is a singular point of $C$, in which case $C$ lies on the divisor $N L$ of curves singular somewhere along $L$. We thus have

$$
\rho^{*} M_{2}=\alpha \cdot T L+\beta \cdot N L \text {. }
$$

The coefficient $\alpha$ in this relation is more or less by definition 1 . To find the coefficient $\beta$, we write down an arc in $W$ meeting $N L$ transversely at a general 
point $C$, and determine the multiplicity of the pullback to this arc of the divisor $M_{2}$. In local coordinates $(x, y)$ in the plane, let $L$ be the line $y=0$, let the node of $C$ lying on $L$ be the origin $x=y=0$, and say the local equation of the curve $C$ is $f_{0}(x, y)=x^{2}-y^{2}=0$. We can then take as our arc the family $\left\{C_{t}\right\}$ given by applying the translations $(x, y) \mapsto(x, y+t)$ to the curve $C$, i.e., in local coordinates

$$
f_{t}(x, y)=x^{2}-(y-t)^{2}=0
$$

since the node of $C_{t}$ is at $(0, t)$ this arc will cross $N L$ transversely at $C=C_{0}$.

To find the multiplicity of $M_{2}$ at 0 , we look at the inverse image in the product $\mathbf{P}^{N} \times \Sigma$ of the arc $\left\{C_{t}\right\}$. We write a point in this inverse image as $\left(f_{t},(p, l)\right)$, where $p$ is the point $(a, b)$ and $l$ the line $(y-b)=m(x-a) ; t, a, b$ and $m$ will serve as coordinates in $U$ near the point $\left(f_{0},((0,0), L)\right)$ (i.e., the point $\left.t=a=b=m=0\right)$. Since $M_{2}$ is defined to be the image of the intersection of $\Psi_{2}$ with the inverse image from $\Sigma$ of the cycle $l=L$, the intersection number of $M_{2}$ with our arc will just be the intersection multiplicity of $\Psi_{2}$ with this cycle at this point, and this is what we shall find. To write down the equations of $\Psi$, observe that in terms of the coordinate $u=x-a$ along the line $l$, we have

$$
\begin{aligned}
f_{t \mid l} & =(u+a)^{2}-(m u+b-t)^{2} \\
& =\left(1-m^{2}\right) \cdot u^{2}+(2 a-2 m b+2 m t) \cdot u+\left(a^{2}-b^{2}+2 b t-t^{2}\right) .
\end{aligned}
$$

The equations of the locus $\Psi_{2}$ in $U$ are then given by setting the coefficients of the linear and constant terms of this polynomial in $u$ equal to zero, i.e., in $U$ the ideal of $\Psi_{2}$ is given by

$$
I_{\Psi_{2}}=\left(a-m b+m t, a^{2}-b^{2}+2 b t-t^{2}\right) .
$$

On the other hand, the equations of the locus $l=L$ are simply $m=b=0$; and on the surface they cut out in $U$, the ideal of $\Psi_{2}$ restricts to $\left(a, t^{2}\right)$. The intersection multiplicity of $\Psi_{2}$ with the cycle $l=L$ in $U$, and hence the coefficient $b$ in (3.6), is thus 2 ; we have

$$
\rho^{*} M_{2} \sim T L+2 N L
$$

We look next at the pullback to the Severi variety of the divisor $M_{3}$ of curves having a point of intersection multiplicity 3 or more with a line of a fixed pencil. Again, the support of this divisor is easy to describe: a curve $C$ having such a point of intersection with $L$ must have either one branch with contact of order at least 3 ; two branches, at least one of which intersects $L$ multiply; or three branches. If we have a codimension one family of such curves in the Severi variety, it must be a component of either $F P, N P$ or $T R$, respectively; so that $\rho^{*} M_{3}$ will be a linear combination of these three divisors. In fact, we claim that

$$
\rho^{*} M_{3} \sim F P+3 N P+6 T R .
$$

Again, the coefficient of $F P$ is one, more or less by definition. We will evaluate the coefficient of $N P$ by the same set-up as above. Specifically, suppose the chosen pencil is the pencil of vertical lines $\{y=\lambda\}$, and let $L$ be the line $y=0$ as above. We want to write down an arc in $W$ meeting $N P$ transversely at a general point $C$; we can suppose that the curve $C$ is given locally as $x\left(y-x^{2}\right)$ and take our arc in 
$W$ to be one obtained simply by applying the family of shears $(x, y) \rightarrow(x, y-t x)$ to the curve $C$. The local equations of the family $\left\{C_{t}\right\}$ will then be

$$
f_{t}(x, y)=x\left(y-t x-x^{2}\right)
$$

since the tangent lines to this family of curves at their common node $x=y=0$ cross the line in $\mathrm{P}^{2^{*}}$ corresponding to the given pencil transversely, the arc $\left\{C_{t}\right\}$ in $W$ will be transverse to $N P$.

As before, we go to the inverse image of our arc in $\mathbf{P}^{N} \times \Sigma$; choosing coordinates as in the previous example, we can write

$$
\begin{aligned}
f_{t \mid l} & =(u+a) \cdot\left(m u+b-t(u+a)-(u+a)^{2}\right) \\
& =-u^{3}+(m-t-3 a) \cdot u^{2}+\left(b-2 t a-3 a^{2}+a m\right) \cdot u+\left(a b-a^{2} t-a^{3}\right),
\end{aligned}
$$

so that the local equations of $\Psi_{3}$ are

$$
m-t-3 a=b-2 t a-3 a^{2}+a m=a b-a^{2} t-a^{3}=0 .
$$

The equation of the pencil $\{y-\lambda\}$, meanwhile, is just $m=0$; solving, we have

$$
t=-3 a ; \quad b=-3 a^{2} .
$$

In other words, the intersection of $\Psi_{3}$ with the locus $m=0$ in the inverse image of our arc lies on the smooth curve defined by the equations $m=0, t=-3 a$ and $b=-3 a^{2}$, and in terms of the local coordinate $a$ on that curve has equations $a^{3}=0$. The intersection multiplicity of our arc with $M_{3}$ is thus 3 .

For the coefficient of $T R$ in (3.8), we choose an arc in $W$ transverse to a general point of $T R$ : take, for example,

$$
f_{t}(x, y)=\left(x^{2}-y^{2}\right) \cdot(x-t)
$$

We get

$$
\begin{aligned}
f_{t \mid l}= & \left((u+a)^{2}-(m u+b)^{2}\right) \cdot(u+a-t) \\
= & \left(1-m^{2}\right) \cdot u^{3}+\left(\left(1-m^{2}\right)(a-t)+(2 a-2 m b)\right) \cdot u^{2} \\
& +\left((2 a-2 m b)(a-t)+a^{2}-b^{2}\right) u+\left(a^{2}-b^{2}\right)(a-t),
\end{aligned}
$$

and after restricting to the locus $m=0$ we have the set of equations for $\Psi_{3}$ :

$$
3 a-t=0, \quad 3 a^{2}-b^{2}-2 a t=0, \quad\left(a^{2}-b^{2}\right)(a-t)=0 .
$$

Restricting further to the smooth surface with equation $t=3 a$, we find the ideal of $\Psi_{3}$ is generated by $3 a^{2}-b^{2}$ and $a\left(a^{2}-b^{2}\right)$; since these are the equations of curves with an ordinary node and an ordinary triple point with no pairwise tangent branches, their intersection number - and correspondingly the coefficient of $T R$ in (3.8)-is 6 .

Next, consider the pullback to $W$ of the divisor $M_{4}$. As usual, the components of the locus of curves $C \in W$ having a point $p$ of intersection multiplicity 4 or more with a line $L$ may be broken up according to the number of branches of a general curve in each component at $p$, and their individual intersection numbers with $L$ : we could have one branch, which would then be a hyperflex; we could have two branches, each tangent to $L$ (i.e., a tacnode); two branches, one with contact of order three (i.e., a flecnode); or three branches, one tangent (i.e., a triple point). 
Lastly, $C$ may actually contain $L$, i.e., lie in $\Delta_{0,1} \cdot \rho^{*} M_{4}$ is thus a linear combination of the divisors $H F, T N, F N, T R$ and $\Delta_{0,1}$; we claim that in fact

$$
\rho^{*} M_{4}=H F+6 T N+4 F N+36 T R+(4 d-12) \Delta_{0,1} .
$$

We will work out explicitly only the coefficients of $T N, F N$ and $T R$ here. The coefficient of $\Delta_{0,1}$ we know only by comparing coefficients in the formula (4.22) of the next chapter; we do not know of a way of computing it directly.

We begin with the coefficient of $T N$; as usual, we start by writing down an arc in $W$ transverse to $T N$ :

$$
f_{t}(x, y)=\left(y-x^{2}+t\right)\left(y+x^{2}-t\right)=y^{2}-x^{4}+2 t x^{2}-t^{2}
$$

(the existence of such a family is guaranteed by the fact that $W$ dominates the equigeneric locus of the versal deformation space of the tacnode of $C_{0}$ ). Next, we restrict to a general line $l$ and expand around a general point on $l$ :

$$
\begin{aligned}
f_{t \mid l}= & (m u+b)^{2}-(u+a)^{4}+2 t(u+a)^{2}-t^{2} \\
= & -u^{4}-4 a \cdot u^{3}+\left(m^{2}-6 a^{2}+2 t\right) \cdot u^{2} \\
& +\left(2 m b-4 a^{3}+4 a t\right) \cdot u+\left(b^{2}-a^{4}+2 t a^{2}-t^{2}\right) .
\end{aligned}
$$

The ideal of $\Psi_{4}$ in the local ring at the point $m=a=b=t=0$ is generated by the relations

$$
\begin{array}{cl}
4 a=0, & m^{2}-6 a^{2}+2 t=0, \\
2 m b-4 a^{3}+4 a t=0, & \text { and } \quad b^{2}-a^{4}+2 t a^{2}-t^{2}=0 .
\end{array}
$$

To find the multiplicity of their intersection, we solve them: from the first equation, $a=0$; and then from the second we have $t=-m^{2} / 2$. In terms of the coordinates $m$ and $b$ on the smooth surface cut out by the first two equations, then, the last two equations read

$$
2 m b=0 \quad \text { and } b^{2}-m^{4} / 4=0 .
$$

The first of these describes of course the two coordinate axes; the second a curve with two branches at the origin, both simply tangent to the $m$-axis, and hence having intersection number 3 with the first curve. The total multiplicity of intersection of our arc $\left\{C_{t}\right\}$ with the divisor $\rho^{*} M_{4}$ at $t=0$-and hence the coefficient of $T N$ in (3.9) -is thus 6.

To do the coefficient of $F N$, we choose a family in $W$ transverse to $F N$ : for example,

$$
f_{t}(x, y)=x\left(y-x^{3}+t x^{2}\right)=x y-x^{4}+t x^{3} .
$$

(The fact that there exists an arc in $W$ with this local equation follows from the fact that the cube of the maximal ideal at the node $(0,0)$ of $C_{0}$ imposes independent conditions on curves of degree $d$ satisfying the adjoint conditions at the other singularities of $C_{0}$.) Restricting to the line $x=u+a, y=m u+b$ as above, we find

$$
\begin{aligned}
f_{t \mid l}= & -u^{4}+(-4 a+t) \cdot u^{3}+\left(m-6 a^{2}+3 a t\right) \cdot u^{2} \\
& +\left(m a+b-4 a^{3}+3 t a^{2}\right) \cdot u+\left(a b-a^{4}+t a^{3}\right) .
\end{aligned}
$$


Solving in sequence the equations we get by setting all but the highest coefficient of the quartic in $u$ equal to zero, we find that the ideal of $\Psi_{4}$ is generated by the relations

$$
t=4 a, \quad m=-6 a^{2}, \quad b=-2 a^{3}, \quad a^{4}=0 .
$$

The multiplicity of this point, and hence the coefficient of $F N$ in (3.9), is thus 4 .

Finally, to find the coefficient of $T R$, we introduce the family $\left\{C_{t}\right\}$ given by

$$
f_{t}(x, y)=\left(x^{2}-y^{2}\right) \cdot\left(y-x^{2}+t\right)
$$

which is transverse to $T R$ at $f_{0}$, and whose existence is assured by the fact that $W$ dominates the equigeneric locus of the versal deformation space of the triple point of $C_{0}$. We have then

$$
\begin{aligned}
F_{t \mid l}= & \left(\left(1-m^{2}\right) u^{2}+2(a-m b) u+\left(a^{2}-b^{2}\right)\right) \cdot\left(-u^{2}+(m-2 a) u+\left(b-a^{2}+t\right)\right) \\
=- & \left(1-m^{2}\right) \cdot u^{4}+\left(-2(a-m b)+\left(1-m^{2}\right)(m-2 a)\right) \cdot u^{3} \\
& +\left(-\left(a^{2}-b^{2}\right)+2(a-m b)(m-2 a)+\left(1-m^{2}\right)\left(b-a^{2}+t\right)\right) \cdot u^{2} \\
& +\left(2(a-m b)\left(b-a^{2}+t\right)+\left(a^{2}-b^{2}\right)(m-2 a)\right) \cdot u+\left(a^{2}-b^{2}\right)\left(b-a^{2}+t\right) .
\end{aligned}
$$

For notation, let $G_{i}$ be the divisor in $U$ defined by the coefficient of $u^{i}$ in this polynomial. To calculate the intersection number $G_{0} G_{1} G_{2} G_{3}$, we introduce the divisors $E^{\prime}=(a-b), E^{\prime \prime}=(a+b), F_{1}=E^{\prime}+E^{\prime \prime}, F_{2}=\left(-a^{2}+b+t\right), F_{3}=(a+m b)$, and $F_{4}=(m-2 a)$. It is not hard to see that the divisors $F_{2}, F_{3}, F_{4}$ and either $E^{\prime}$ or $E^{\prime \prime}$ intersect transversely, so that the intersection number $F_{1} F_{2} F_{3} F_{4}=2$. We have then

$$
G_{0}=F_{1}+F_{2}
$$

and since $G_{1} \equiv F_{2}+F_{3} \bmod F_{1}$, and $G_{1} \equiv F_{1}+F_{4} \bmod F_{2}$, we have

$$
G_{0} G_{1}=2 F_{1} F_{2}+F_{1} F_{3}+F_{2} F_{4} .
$$

Similarly, we arrive at

$$
G_{0} G_{1} G_{2}=3 F_{1} F_{2} F_{3}+3 F_{1} F_{2} F_{4}
$$

and

$$
G_{0} G_{1} G_{2} G_{3}=6 F_{1} F_{2} F_{3} F_{4}=12 .
$$

Finally, since the line $L$ is one of three lines having intersection number 4 with the curve $C$ given by $f_{0}$, the total multiplicity of the divisor $\rho^{*} M_{4}$ on our arc is 36 .

Lastly, the pullback of $N_{3}$ to $W$ : it is not hard to see that a codimension one locus of curves $C \in W$ having intersection number three or more with a line at some point of a line $L$ must consist of curves either (a) having a genuine flex somewhere on $L$, i.e., lying in $F L$; (b) having a node somewhere on $L$, i.e., lying in $N L$; or (c) containing a line, i.e., lying in $\Delta_{0,1}$. In fact, we have

$$
\rho^{*} N_{3}=F L+6 N L+\Delta_{0,1} .
$$

We will omit the verification of the multiplicities, out of a combination of consideration for the reader and plain laziness (actually, we omit only the verification of the coefficient of $N L$, since the coefficient of $F L$ is 1 by definition and the coefficient of $\Delta_{0,1}$ comes only from comparison of the above formula for $\rho^{*} N_{3}$ with formulas (4.3), (4.14) and (4.15)). 
4. Further computations of divisor classes. In this chapter we will make some further computations of classes of the divisors introduced in $\S 1$, combining all the various techniques we have at our disposal. We will derive some of these classes several times over, a duplication that will allow us to verify that the coefficients of $\Delta$ given in formulas (1.1)-(1.3) are correct.

The techniques that we will use fall roughly into three categories. First, we can describe all the loci we will be dealing with as degeneracy loci associated to some map of vector bundles, and apply Porteous' formula or a specialization to find its class, as in $\S 2$ above. Second, we have the relations obtained in the last chapter. Third, we have various techniques relating to the characteristic classes of the loci themselves: we have the adjunction formula in several guises, as well as the Riemann-Hurwitz formula for branched covers and the double point formula.

(a) Nodes. We begin by computing the class of the divisor $N \subset \mathscr{C}$ of points lying over assigned nodes of the images of their fibers. Probably the simplest way to do this is via the double point formula: since $N$ is just the double point locus of the map $\varphi$, we have

$$
\begin{aligned}
\omega_{\mathscr{C} / W} & =\varphi^{*} \omega_{S / W} \otimes \mathscr{O}(-N)=\varphi^{*}\left(\omega_{\mathbf{P}^{2} \times W / W}(S)\right) \otimes \mathscr{O}(-N) \\
& \sim(d-3) \cdot D+\pi^{*} A-N,
\end{aligned}
$$

from which we deduce that

$$
N \sim(d-3) \cdot D-\omega+\pi^{*} A .
$$

(To give a more concrete version of this argument, we can write down a meromorphic global section of the relative dualizing sheaf: let $x$ and $y$ be euclidean coordinates in the plane $\mathbf{P}^{2}$, and $\lambda=\left(\lambda_{1}, \ldots, \lambda_{N}\right)$ euclidean coordinates on $\mathbf{P}^{N}$, and set

$$
\Psi=\varphi^{*} \frac{d x}{\partial f_{\lambda} / \partial y}
$$

We see that the zeroes and poles of $\Psi$ consist of a zero of order $d-3$ along the line at $\infty$ in $\mathbf{P}^{2}$, a simple pole along the divisor $N$, and a simple zero along the hyperplane at $\infty$ in $\mathbf{P}^{N}$; formula (4.1) follows.

(b) Nodes II. We can also calculate the class of $N$ in the manner of $\S 2$, as follows. We will use the notation of $\S 2$; in particular, recall that $\mathscr{C}_{2}$ is the fiber product $\mathscr{C} \times{ }_{W} \mathscr{C}$ of $\mathscr{C}$ with itself over $W$, and that $\mathscr{F}_{(1,1)}$ is the bundle over $\mathscr{C}_{2}$ whose fiber at a point $(p, q)$ is the space of sections of $\mathscr{L}_{C} / \mathscr{L}_{C}(-p-q)$. We have a natural evaluation map from the trivial bundle $\mathscr{E}=H^{0}\left(\mathbf{P}^{2}, \mathscr{O}(1)\right) \otimes \mathscr{O}_{\mathscr{C}}$ to the bundle $\mathscr{F}_{(1,1)}$, and the closure $\Phi$ of the locus of pairs $(p, q)$ of distinct points of a fiber of $\pi: \mathscr{C} \rightarrow W$ mapping to the same point of $\mathbf{P}^{2}$ will just be the locus in $\mathscr{C}_{2}$ where this map fails to have rank 2. The class of $\Phi$ is then given as the second Segre class of the bundle $\mathscr{F}_{(1,1)}$, and so we have

$$
\begin{aligned}
{[\Phi] } & =c_{1}\left(\mathscr{F}_{(1,1)}\right)^{2}-c_{2}\left(\mathscr{F}_{(1,1)}\right) \\
& =D_{1}^{2}+D_{2}^{2}+2 D_{1} \cdot D_{2}-\omega_{1} \cdot \Delta_{12}-4 D_{1} \cdot \Delta_{12}-D_{1} \cdot D_{2}+D_{1} \cdot \Delta_{12} \\
& =D_{1}^{2}+D_{2}^{2}+D_{1} \cdot D_{2}-\omega_{1} \cdot \Delta_{12}-3 D_{1} \cdot \Delta_{12} .
\end{aligned}
$$

Now, $\pi_{1 . *} D_{1}^{2}=0$, while $\pi_{1 .} D_{2}^{2}=\pi^{*} A$ and $\pi_{1 *} D_{1} \cdot D_{2}=d \cdot D$; so the pushforward of $[\Phi]$ to $\mathscr{\mathscr { C }}$ is

$$
[N]=\pi_{1 .}[\Phi]=(d-3) D-\omega+\pi^{*} A
$$

as we had previously calculated. 
Note further that we did not have to exclude from this calculation the inverse image of $\Delta$, since the sheaf $\mathscr{F}_{(1,1)}$ will fail to be a locally free sheaf with fiber as specified above only over a codimension 2 locus in $\mathscr{C}$, namely the locus of points singular in their fibers. The formula (4.1) is thus established over all of $\mathscr{C}$ by this computation.

(c) Nodes on a line. We look next at the divisor $N L$ of curves having an assigned node on a given line $L \subset \mathbf{P}^{2}$. The class of $N L$ follows immediately from (4.1): the locus of curves with a node along the line $L$ is just the image in $W$ of the intersection $\eta^{*} L \cap N$; since $\pi$ maps this intersection onto its image with degree 2 , we have

$$
\begin{aligned}
N L & =\pi_{*}(D \cdot N) / 2=\pi_{*}\left(D \cdot\left((d-3) D-\omega+\pi^{*} A\right)\right) / 2 \\
& =\pi_{*}\left((d-3) D^{2}-D \cdot \omega\right) / 2+d \cdot A / 2 \\
& =\frac{2 d-3}{2} A-\frac{1}{2} B
\end{aligned}
$$

(d) Cusps II. There is an alternate way to calculate the class of the divisor $C U$, and one that allows us to determine the coefficients of the boundary components $\Delta$ at the same time. This is to look at the map $\varphi: \mathscr{C} \rightarrow W \times \mathbf{P}^{2}$, and to observe that $C U$ is just the image in $W$ of the locus of points in $\mathscr{C}$ where the differential $d \varphi$ fails to have maximal rank 2. By Porteous' formula, then,

$$
C U=\pi_{*}\left(\left[\eta^{*} c\left(\Theta_{W \times \mathbf{P}^{2}}\right) / c\left(\Theta_{\mathscr{f}}\right)\right]_{2}=\pi_{*}\left(\left[\varphi^{*} c\left(\Theta_{\mathbf{P}^{2}}\right) \pi^{*} c\left(\Theta_{W}\right) / c\left(\Theta_{\mathscr{C}}\right)\right]_{2}\right) .\right.
$$

From the exact sequence

$$
0 \rightarrow \pi^{*} \Omega_{W} \rightarrow \Omega_{\digamma} \rightarrow \omega_{\leftarrow / W} \otimes \mathscr{I}_{\delta} \rightarrow 0
$$

(where $\delta$ is the locus of singular points of fiber in $\mathscr{C}$ ) we see that

$$
c\left(\Theta_{\digamma}\right)=\pi^{*} c\left(\Theta_{W}\right) \cdot(1-\omega+[\delta]),
$$

so that

$$
\pi^{*} c\left(\Theta_{W}\right) / c\left(\Theta_{\leftarrow}\right)=1+\omega+\omega^{2}-[\delta] .
$$

Thus

$$
\begin{aligned}
C U & =\pi_{*}\left(\left[\left(1+3 D+3 D^{2}\right) \cdot\left(1+\omega+\omega^{2}-[\delta]\right)\right]_{2}\right) \\
& =\pi_{*}\left(3 D^{2}+3 D \omega+\omega^{2}-[\delta]\right) \\
& =3 A+3 B+C-\Delta .
\end{aligned}
$$

Observe that we have now verified formula (1.1) over all of $W$.

(e) Tangent to a line. We look next at the class of the divisor $T L \subset Z$ of curves tangent to a fixed line $L$ (precisely, we mean the closure of the locus of curves $C$ with a smooth point $p \in C$ tangent to $L$; in particular, we do not mean to include the divisor $N L$ of curves with a node somewhere along $L$ ). We can find its class, modulo the boundary components $\Delta$, in straightforward fashion: the line $L$ is the zero locus of a section $\sigma \in H^{0}\left(\mathbf{P}^{2}, \mathscr{O}(1)\right)$, which in turn defines by evaluation a section $\tilde{\sigma}$ of the bundle $\mathscr{F}_{(2)}$ introduced in $\S 2$ whose fiber over a point $p \in \mathscr{C}$ will be the space of sections of $\mathscr{L}_{C} / \mathscr{L}_{C}(-2 p)$. The locus $\Theta \subset \mathscr{C}$ of points of tangency of the curves in $W$ with $L$ will be the zero locus of this section of $\mathscr{F}_{(2)}$, and the divisor $T L$ the image of $\Theta$ in $W$. We recall from $\S 2$ that

$$
c\left(\mathscr{F}_{(2)}\right)=(1+D) \cdot(1+D+\omega)
$$


so that $c_{2}\left(\mathscr{F}_{(2)}\right)=D^{2}+D \cdot \omega$ and

$$
T L \sim \pi_{*}\left(D^{2}+D \cdot \omega\right)=A+B
$$

modulo the boundary components $\Delta$.

(f) Tangent to a line II. There is another approach to computing the class of $T L$, and one that allows us to determine the coefficients of the boundary components $\Delta$ as well. This is by observing that the divisor $T L$ is just the branch divisor of the map from $\eta^{-1}(L)$ to $W$, and using the adjunction formula to determine the class of the ramification divisor of this map. Since $\eta^{-1}(L) \sim D$, the adjunction formula gives us

$$
T L \sim \pi_{*}((D \cdot(D+\omega)))=\pi_{*}\left(D^{2}+D \omega\right)=A+B .
$$

(g) Tangent to a line III. A third way to calculate the class of $T L$ is to use the formulas of the preceding chapter. Specifically, we have seen that the divisor $M_{2}$ in $\mathbf{P}^{2}$ of curves having a multiple point of intersection with $L$ is a hypersurface of degree $2 d-2$; its pullback to the Severi variety $W$ is thus a divisor of class $(2 d-2) \cdot A$. But we also saw in the last chapter that this pullback is just the sum of the divisors $T L$ and $2 \cdot N L$; we have accordingly

$$
T L \sim(2 d-2) \cdot A-2 \cdot N L \sim A+B .
$$

(h) Tangent to a pencil. Next, we fix a point $p \in \mathbf{P}^{2}$ and let $T \subset \mathscr{C}$ be the closure of the locus of points $q$ such that the tangent line to the image $\eta(C)$ of the fiber of $\pi$ through $q$ passes through $p$. The standard way of finding the class of the divisor $T$ is to view the pencil of lines through $p$ as a two-dimensional subspace $V$ of $H^{0}\left(\mathbf{P}^{2}, \mathscr{O}(1)\right)$ and looking at the corresponding map from the trivial bundle $\mathscr{E}^{\prime}=V \otimes \mathscr{O}_{\leftarrow}$. to the bundle $\mathscr{F}_{(2)} . T$ will then be the locus where this map fails to be an isomorphism, and so

$$
T \sim c_{1}\left(\mathscr{F}_{(2)}\right)=2 D+\omega .
$$

Note that in the above computation, we did not actually have to exclude the boundary $\Delta$, since the sheaf $\mathscr{F}_{(2)}$ will fail to be a well defined, locally free sheaf only in codimension 2 . Thus this formula is in fact valid over all of $\mathscr{C}$.

(i) Tangent to a pencil II. Another way to evaluate the class of the divisor $T$ on $\mathscr{C}$ is to view $T$ as the ramification divisor of the map $\mathscr{C} \rightarrow W \times \mathbf{P}^{1}$ defined by composing $\varphi$ with projection from the point $p$ in each fiber of $W \times \mathbf{P}^{2}$. (This projection map is course only a rational map, since it fails to be well defined at points of $\eta^{-1}(p)$; but since this locus has codimension 2 and we are concerned with a divisor class on $\mathscr{C}$ this should not bother us.) By Riemann-Hurwitz, then, we have

$$
T=c_{1}\left(\omega_{\leftarrow / W}\right)-c_{1}\left(\omega_{W \times \mathbf{P}^{1} / W}\right)=\omega+2 D,
$$

as before.

(j) Nodes tangent to a pencil. One consequence of the calculation of $[T]$ is that we can describe the divisor $N P$ on $W$, where $N P$ is the closure of the locus of curves $C$ such that the tangent line to a branch of $\eta(C)$ at a node passes through a given point $p \in \mathbf{P}^{2}$. This would be just the image in $W$ of the intersection of the locus $T$ of points where the tangent line to $\eta(C)$ at $q$ passes through $p$ with the locus 
$N$ of points lying over nodes, except for one thing: $T$ and $N$ also intersect in the locus of points lying over cusps (a local computation shows that this intersection is transverse). Now, we have

$$
\begin{aligned}
\pi_{*}(F \cdot N) & \sim \pi_{*}\left((\omega+2 D) \cdot\left((d-3) D-\omega+\pi^{*} A\right)\right) \\
& =\pi_{*}\left((2 d-6) D^{2}+(d-5) \omega D-\omega^{2}+(\omega+2 D) \cdot \pi^{*} A\right) \\
& =(2 d-6) A+(d-5) B-C+(2 d+2 g-2) A \\
& =(4 d+2 g-8) A+(d-5) B-C
\end{aligned}
$$

and hence

$$
\begin{aligned}
N P & \sim \pi_{*}(F \cdot N)-C U \\
& =(4 d+2 g-11) A+(d-8) B-2 C+\Delta .
\end{aligned}
$$

(k) Flexes. Consider next the divisor $F$ on $\mathscr{C}$ of points lying over flexes of the corresponding plane curves. To determine the class of this divisor, we set up the bundle $\mathscr{F}_{(3)}$ on $\mathscr{C}$, whose fiber over a point $p \in \mathscr{C}$ will be the space of sections of $\mathscr{L}_{C} / \mathscr{L}_{C}(-3 p)$; we will then have a map $\varphi: \mathscr{E} \rightarrow \mathscr{F}_{(3)}$ defined by evaluation, and the divisor of flexes will just be the locus where $\varphi$ is singular. Its class is thus the first Chern class of the bundle $\mathscr{F}_{(3)}$. To compute this class, observe that we have a filtration of $\mathscr{F}_{(3)}$ given by the successive quotients

$$
\mathscr{F}_{(3)} \rightarrow \mathscr{F}_{(2)} \rightarrow \mathscr{F}_{(1)} \rightarrow 0,
$$

where $\mathscr{F}_{(k)}$ is the bundle with fiber $\mathscr{L}_{C} / \mathscr{L}_{C}(-k p)$ at $p$. The successive quotients of this filtration are $\mathscr{L}, \mathscr{L} \otimes \omega$, and $\mathscr{L} \otimes \omega^{2}$, so that

$$
F \sim c_{1}\left(\mathscr{F}_{(3)}\right)=3 D+3 \omega,
$$

modulo the boundary $\Delta$.

As before, note that we did not actually have to exclude the boundary $\Delta$ in this computation, since the sheaf $\mathscr{F}_{(3)}$ will fail to be a well defined, locally free sheaf only codimension 2 . There is, however, one slight hitch in this case: over a point of the boundary component $\Delta_{0,1}$ - that is, when the fiber $C$ of $\mathscr{C}$ is reducible, with one component $C_{0}$ mapping to a line and the other component $C_{1}$ to a nodal plane curve of degree $d-1$ and genus $g$ - the determinant of $\varphi$ will vanish identically along $C_{0}$. To see to what order it vanishes along $C_{0}$, observe that as we approach the nodal curve, a total of 3 flexes of the nearby smooth curves tend toward the node; the locus $F$ will consist of a single smooth arc simply tangent to the branch of the node lying on $C_{1}$ and covering $W$ with degree 3 (c.f. [Eisenbud-Harris 2]). In particular, we see that the intersection number of $F$ with $C_{0}$ is exactly 1 . Now, if we write

$$
F=3 D+3 \omega-m \tilde{\Delta}_{0,1}
$$

(recall that $\tilde{\Delta}_{0,1}$ is the union of the components of fibers over $\Delta_{0,1}$ mapping to lines) then the intersection number of $F$ with $C_{0}$ is

$$
\left(F \cdot C_{0}\right)=3\left(D \cdot C_{0}\right)+3\left(\omega \cdot C_{0}\right)-m\left(\tilde{\Delta}_{0,1} \cdot C_{0}\right)=m
$$

from which of course we conclude that $m=1$, i.e., that

$$
F \sim 3 D+3 \omega-\tilde{\Delta}_{0,1} .
$$


(l) Flecnodes. The above calculation allows us to determine as well the class of the divisor $F N \subset W$ of curves possessing a flecnode, that is, a node at which one of the branches is a flex point. To do this, consider the intersection of the locus $F$ with the divisor $N$. This intersection will have two components: the locus of points lying over flecnodes of the corresponding plane curves, and the locus of points lying over cusps. Moreover, the latter occurs with multiplicity two, since both the divisors $F$ and $N$ are ramified along the locus of points lying over cusps (in the case of $N$, this is just the local picture given in $\S 1$; for the description of $F$ near $C U$, see [Eisenbud-Harris 1]). Since we have

$$
N \sim(d-3) \cdot D-\omega+\pi^{*} A .
$$

this gives us

$$
\begin{aligned}
F N \sim & \pi_{*}(F \cdot N)-2 C U \\
= & \pi_{*}\left(\left(3 D+3 \omega-\tilde{\Delta}_{0,1}\right) \cdot\left((d-3) D-\omega+\pi^{*} A\right)\right) \\
& -6 A-6 B-2 C+2 \Delta \\
= & 3(d-3) A+((3(d-3)-3) B-3 C+3 d A+3(2 g-2) A \\
& -(d-3) \Delta_{0,1}-\Delta_{0,1}-6 A-6 B-2 C+2 \Delta \\
= & (6 d+6 g-21) A+(3 d-18) B-5 C+2 \Delta-(d-2) \Delta_{0,1} .
\end{aligned}
$$

(m) Flexes on a line. We can also use the computation of the class of the divisor $F$ of flexes to describe the divisor $F L \subset W$ of curves having a flex point on a fixed line $L \subset \mathrm{P}^{2}: F L$ will just be the image of the intersection of the divisor $\eta^{*} L \sim D$ with the locus $F$, so that we have

$$
\begin{aligned}
F L & \sim \pi_{*}(F L \cdot D) \\
& =\pi_{*}\left(3 D^{2}+3 \omega D-\tilde{\Delta}_{0,1} D\right) \\
& =3 A+3 B-\Delta_{0,1} .
\end{aligned}
$$

(n) Flexes on a line II. As usual, an alternate approach to determining the class of the divisor $F L$ is to look at the divisor $N_{3}$ on $\mathbf{P}^{N}$ consisting of curves with a flex along the line $L$, and describe its restriction to $W$. What we saw in the last chapter is that $N_{3}$ pulls back to the divisor $F L$, plus six times the divisor $N L$, plus the divisor $\Delta_{0,1}$. We thus have

$$
(6 d-6) A \sim F L+6 \cdot N L+\Delta_{0,1},
$$

so that

$$
\begin{aligned}
F L & \sim(6 d-6) A-3(2 d-3) A+3 B-\Delta_{0,1} \\
& =3 A+3 B-\Delta_{0,1} .
\end{aligned}
$$

(o) Flex lines in a pencil. The divisor we will look at is the closure $F P$ of the locus of curves $C$ that have contact of order 3 or more with a line of a fixed pencil, at a smooth point of $C$. As in the case of the divisor $F L$ above, there are several ways to approach this; in the end, comparing the results will allow us to determine the coefficient of the boundary component $\Delta$ in the divisor $T R$.

The standard way to determine the class of $F P$ is, as in the case of the divisor $T$ discussed in $(4 h)$ above, to introduce the trivial subbundle $\mathscr{E}^{\prime} \subset \mathscr{E}$ coming from 
the subspace $V \subset H^{0}\left(\mathbf{P}^{2}, \mathscr{O}(1)\right)$ corresponding to the given pencil, and look at the bundle map $\varphi: \mathscr{E}^{\prime} \rightarrow \mathscr{F}_{(3)}$ given by evaluation. $F P$ will then be the locus where the map $\varphi$ fails to have rank 2 , from which we conclude (using information from $\S 4(\mathrm{k})$ above) that

$$
\begin{aligned}
F P & \sim \pi_{*}\left(c_{2}\left(\mathscr{F}_{(3)}\right)\right. \\
& =\pi_{*}\left(3 D^{2}+6 \omega D+2 \omega^{2}\right) \\
& =3 A+6 B+2 C,
\end{aligned}
$$

modulo the boundary components $\Delta$.

(p) Flex lines in a pencil II. A second approach to the class of $F P$ is to look at the branching of the divisor $T$ in $\mathscr{C}$ over $W$. For any fiber $C$ of $\pi$, the composition of the map $\eta: C \rightarrow \mathbf{P}^{2}$ with the projection to a line from a point $p \in \mathbf{P}^{2}$ will have exactly $2 d+2 g-2$ simple ramification points, unless either $C$ is singular, or $C$ contains $p$, or $C$ has a flex passing through $p$. The branch divisor $B R_{T}$ of the covering $\pi: T \rightarrow W$ thus consists of the sum of multiples of the divisors $\Delta, A$ and $F P$; and it is not hard to see the multiplicity in all three cases is one. On the other hand, by the adjunction formula the class of the ramification divisor of $T$ over $W$ is just the restriction to $T$ of the sum $T+\omega$; so that $B R_{T}$ has class

$$
\begin{aligned}
B R_{T} & \sim \pi_{*}(T \cdot(T+\omega)) \\
& =\pi_{*}((\omega+2 D) \cdot(2 \omega+2 D)) \\
& =4 A+6 B+2 C .
\end{aligned}
$$

We thus have

$$
F P \sim B R_{T}-A-\Delta \sim 3 A+6 B+2 C-\Delta .
$$

(q) Flex lines in a pencil III. There is a third way to evaluate the class of $F P$, which is to consider the pullback to $W$ of the locus $M_{3} \subset \mathbf{P}^{N}$ of curves of degree $d$ having a point of intersection multiplicity 3 or more with a line $L$ of the given pencil. We have seen that the restriction of $M_{3}$ to $W$ is the linear combination $F P+3 N P+6 T R ;$ thus

$$
\left(3 d^{2}-6 d\right) A \sim F P+3 N P+6 T R
$$

and so

$$
\begin{aligned}
F P \sim & \left(3 d^{2}-6 d\right) A-3 N P-6 T R \\
= & \left(3 d^{2}-6 d\right) A-3((4 d+2 g-11) A+(d-8) B-2 C+\Delta) \\
& -6\left(\left(\left(d^{2}-6 d+8\right) / 2-g+1\right) A-(d-6) B / 2+2 C / 3-\Delta / 3\right) \\
= & 3 A+6 B+2 C-\Delta .
\end{aligned}
$$

We observe that, comparing this third computation on the class of $F P$ with the second, we deduce that the coefficient of $\Delta$ in the formula (1.3) for the class of $T R$ is correct.

(r) Hyperflexes. The calculations above also allow us to determine the class of the divisor $H F \subset Z$ of curves with a hyperflex. Observe first that this can be done modulo the boundary components $\Delta$ in the standard way: away from the boundary $\Delta$, we introduce the vector bundle $\mathscr{F}_{(4)}$ on $\mathscr{C}$ whose fiber at a point $p$ lying on the fiber $C$ of $\pi$ is the space of sections of $\mathscr{L}_{C} / \mathscr{L}_{C}(-4 p)$; we have a natural evaluation 
map $\varphi: \mathscr{E} \rightarrow \mathscr{F}_{(4)}$, and the locus $\Theta$ of hyperflexes will be the locus where $\varphi$ has rank 2. We compute, in the same manner as in $\S 4(\mathrm{k})$ above, that

$$
\begin{aligned}
c\left(\mathscr{F}_{(4)}\right) & =(1+D)(1+D+\omega)(1+D+2 \omega)(1+D+3 \omega) \\
& =1+(4 D+6 \omega)+\left(6 D^{2}+18 D \omega+11 \omega^{2}\right)+\cdots
\end{aligned}
$$

and so

$$
[\Theta]=c_{2}\left(\mathscr{F}_{(4)}\right)=\left(6 D^{2}+18 D \omega+11 \omega^{2}\right) .
$$

Pushing forward to $W$, we find that

$$
H F \sim 6 A+18 B+11 C
$$

modulo the boundary components $\Delta$.

(s) Hyperflexes II. The reader will not be overly surprised at this point to hear that there is another way of calculating the class of $H F$. We consider the curve $F \subset \mathscr{C}$ introduced above, and try to determine the class of the branch divisor $B R_{F}$ of $F$ over $W$. We can do this in two ways: first, since we know the class of $F$ on $\mathscr{C}$, we can use the adjunction formula. We have

$$
F \sim 3 D+3 \omega-\tilde{\Delta}_{0,1}
$$

and so

$$
\begin{aligned}
B R_{F} & =\pi_{*}(F \cdot(F+\omega)) \\
& =\pi_{*}\left(\left(3 D+3 \omega-\tilde{\Delta}_{0,1}\right) \cdot\left(3 D+4 \omega-\tilde{\Delta}_{0,1}\right)\right) \\
& =9 A+21 B+12 C .
\end{aligned}
$$

On the other hand, we know from our local pictures exactly where and how $F$ ramifies over $W$. To begin with, it is unramified over a general point of the divisors $T N$ and $T R$. Next, when a node of a plane curve turns into a cusp, we see [Eisenbud-Harris 1] that two flexes come into the cusp and are simply ramified. Over a point of $\Delta$ the situation is more complicated. Excluding for the moment the component $\Delta_{0,1}$ of $\Delta$, the picture is this: a total of six flexes of the nearby smooth curves will approach the node, comprising two smooth arcs. Each arc will be simply tangent to one of the branches of the fiber $C$ over $\Delta$, hence will have intersection number 3 with $C$, hence will be a three-sheeted cover of $W$ totally ramified over $\Delta$, as depicted in Figure 6 (see [Cukierman] for an analysis of this situation). The situation is different only for the boundary component $\Delta_{0,1}$; here, only three flexes approaches the node, forming a single smooth arc simply tangent to the component $C_{1}$ and hence totally ramified of degree 3 over $\Delta_{0,1}$. finally, the divisor $F$ will be simply ramified along the locus of hyperflexes.

In sum, we see from this description that the branch divisor of $F$ over $W$ is

$$
B R_{F} \sim C U+H F+6\left(\Delta-\Delta_{0,1}\right)+2 \Delta_{0,1} .
$$

Thus

$$
\begin{aligned}
H F & \sim B R_{F}-C U-6 \Delta+4 \Delta_{0,1} \\
& \sim(9 A+21 B+12 C)-(3 A+3 B+C-\Delta)-6 \Delta+4 \Delta_{0,1} \\
& \sim 6 A+18 B+11 C-5 \Delta+4 \Delta_{0,1} .
\end{aligned}
$$




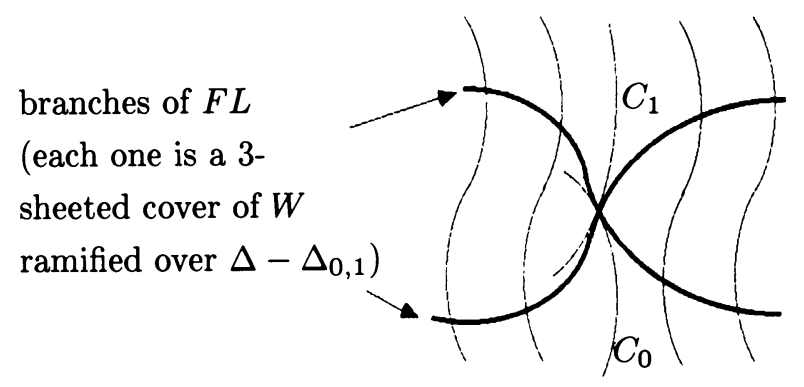

FIGURE 6

(t) Hyperflexes III. There is yet a third way to determine the class of $H F$ (at least modulo $\Delta_{0,1}$ ), by restricting to the Severi variety the divisor $M_{4}$ of curves in $\mathbf{P}^{2}$ that have a point of intersection multiplicity 4 with some line. Recall that we have

$$
M_{4}=H F+6 T N+4 F N+36 T R+(4 d-12) \Delta_{0,1}
$$

(in fact, we determined in $\S 3(\mathrm{~b})$ only the first four coefficients; the coefficient of $\Delta_{0,1}$ will follow from the computation below) while, on the other hand, we know that the restriction to $W$ of the divisor $M_{4}$ is linearly equivalent to $\left(18 d^{2}-78 d+36\right) \cdot A$. Thus

$$
\begin{aligned}
H F \sim & \left(18 d^{2}-66 d+36\right) A-6 T N-4 F N-36 T R-(4 d-12) \Delta_{0,1} \\
\sim & \left(18 d^{2}-66 d+36\right) A-(4 d-12) \Delta_{0,1} \\
& -36\left(\left(\left(d^{2}-6 d+8\right) / 2-g+1\right) A-(d-6) B / 2+2 C / 3-\Delta / 3\right) \\
& -6((3(d-3)+2 g-2) A+(d-9) B-5 C / 2+3 \Delta / 2) \\
& -4\left((6 d+6 g-21) A+(3 d-18) B-5 C+2 \Delta-(d-2) \Delta_{0,1}\right) \\
= & 6 A+18 B+11 C-5 \Delta+4 \Delta_{0,1} .
\end{aligned}
$$

(u) The branch divisor of $N$. The last divisor we shall consider is the branch locus $B R_{N}$ of the covering $\pi: N \rightarrow W$. We will be able to find its class in two different ways, which in turn will allow us to deduce the coefficient of the boundary components $\Delta$ in the formula (1.2) for the class of $T N$.

The first approach to describing $B R_{N}$ is by the adjunction formula: we have

$$
\begin{aligned}
B R_{N} & =\pi_{*}(N \cdot(N+\omega)) \\
& =\pi_{*}\left(\left((d-3) D-\omega+\pi^{*} A\right) \cdot\left((d-3) D+\pi^{*} A\right)\right) \\
& =\pi_{*}\left((d-3)^{2} D^{2}-(d-3) \omega D+((2 d-6) D-\omega) \pi^{*} A\right) \\
& =\left((d-3)^{2}+(2 d-6) d-(2 g-2)\right) A-(d-3) B \\
& =\left(3 d^{2}-12 d+9-(2 g-2)\right) A-(d-3) B .
\end{aligned}
$$

(v) The branch divisor of $N$ II. The other way we have of evaluating the class of $B R_{N}$ is by direct examination: we can explicitly describe the divisor $B R_{N}$ as a linear combination of the divisors studied so far. Specifically, a curve $\eta(C) \in W$ will have fewer than $\delta$ distinct assigned nodes if and only if it has either a tacnode or a triple point, and there will be fewer than $2 \delta$ points of $C$ lying over these nodes if and only if one of them is a cusp; so that $B R_{N}$ must be a linear combination 
of the divisors $C U, T N$ and $T R$. From the local descriptions given in $\S 1$ above, we see that $N$ is simply ramified over $C U$, has two simple ramification points over $T N$, and has three nodes lying over a point of $T R$; thus

$$
\begin{aligned}
B R_{N} \sim & 6 T R+2 T N+C U \\
\sim & \left.6\left(\left(d^{2}-6 d+8\right) / 2-g+1\right) A-(d-6) B / 2+2 C / 3-\Delta / 3\right) \\
& +2((3(d-3)+2 g-2) A+(d-9) B-5 C / 2+3 \Delta / 2) \\
& +3 A+3 B+C-\Delta \\
= & \left(\left(3 d^{2}-12 d+9\right)-(2 g-2)\right) A-(d-3) B
\end{aligned}
$$

as before. Again, since we have already verified the coefficients of $\Delta$ in formulas (1.1) and (1.3) for the classes of $C U$ and $T R$, this establishes the coefficient of $\Delta$ in the formula (1.2) for $T N$, and completes our proof of these formulas.

(w) Pullbacks of divisors from the Hilbert scheme. As we defined it in $\S 1$, the Severi variety $W$ lives in the product of $\mathbf{P}^{N}$ with the Hilbert scheme $\mathscr{H}$ of subschemes of dimension zero and degree $\delta$ in $\mathbf{P}^{2}$. We can ask, then, whether there are any interesting new divisor classes among those pulled back to $W$ from $\mathscr{H}$.

The answer is no. The Picard group of $\mathscr{H}$ is generated by two classes: there is the class $\alpha$ of the locus of subschemes whose support intersects a line $L \subset \mathbf{P}^{2}$ (that is, the image in $\mathscr{H}$ of the intersection of the universal subscheme in $\mathscr{H} \times \mathbf{P}^{2}$ with the pullback $\mathscr{H} \times L$ of $L$ ), and the class $\beta$ of the locus of nonreduced subschemes (cf. [Ellingsrud-Stromme]). The pullbacks of these classes to $W$ are clear: $\alpha$ pulls back to the divisor $N L$, while $\beta$ pulls back to the branch divisor $B R_{\bar{N}}$ of $\bar{N}$ over $W$. From the local picture of the locus $\bar{N}$ given is $\S 1$, then, we have

$$
\Phi^{*} \alpha \sim(2 d-3) A / 2-B / 2, \quad \text { and } \quad \Phi^{*} \beta \sim T N+4 T R
$$

(the coefficient 4 of $T R$ in this last equation comes from the fact that the locus of nodes has a spatial triple point, as shown in Figure 5).

5. The canonical class of the Severi variety. In this section we will consider a collection of vector bundles living on the Severi variety $W$, which we may call the adjoint bundles. These are simply the bundles $E_{n}$ on $W$ whose fiber at a point $C \in W$ is just the adjoint series of degree $n$ on the plane curve $C$ with respect to its assigned singularities, that is, the vector space of polynomials of degree $n$ satisfying the adjoint conditions of $C$ at the assigned singularities. Officially, it may be defined in terms of the spaces and maps of the diagram on page 6 as the direct image $\pi_{1 .}\left(\pi_{2}^{*} \mathscr{O}_{\mathbf{P}^{2}}(d) \otimes \mathscr{I}_{\bar{N}}\right)$, where $\pi_{1}, \pi_{2}$ are the projection maps on $\mathbf{P}^{2} \times Z$. As we will see below this bundle, modulo the subbundle with fiber at $C$ the space of polynomials vanishing on $C$, can also be characterized as the pushforward, from the universal curve $\mathscr{C} \rightarrow W$ of arithmetic genus $g$ over $W$, of the line bundle $\omega \otimes \mathscr{L}^{n-d+3} \otimes \pi^{*} \mathscr{O}(-A)$. Each characterization of $E_{n}$ give us a way of calculating its Chern classes, at least when $n \geq d-3$, and we carry this out here.

One of the bundles $E_{n}$ is of particular interest: by virtue of the description [Diaz-Harris] of the tangent space to the space of equigeneric deformations of a plane curve singularity, the bundle $E_{d}$ is closely related to the tangent bundle of the Severi variety itself. In the latter half of this chapter, we will describe that relationship, and use it to determine the canonical class of $W$. 
(a) Classes of the adjoint bundles via Riemann-Roch. To begin with, the classes of the bundle $E_{n}$ are readily calculated for $n \geq d-3$ by the Grothendieck-RiemannRoch formula. First, we have the formula

$$
\omega=(d-3) \cdot D-N+\pi^{*} A
$$

established above ((4.1)). Next, recall that the curves of degree $n$ satisfying the adjoint conditions at a subset of the singularities of a reduced plane curve of degree $d$ cut out the complete linear series $\omega_{C}(n-d+3)$ on the normalization $C$ of the curve at those singularities. It follows that we have for any $n$ a surjection

$$
E_{n} \rightarrow\left(\pi_{*}\left(\omega_{\mathscr{C} / W} \otimes \mathscr{L}^{n-d+3}\right)\right) \otimes \mathscr{O}_{W}(-A) .
$$

The kernel $F_{n}$ of this map is the bundle whose fiber at every point $C \in W$ is just the space of polynomials of degree $n$ vanishing on the curve $C$; it is thus a tensor product of the trivial bundle $H^{0}\left(\mathbf{P}^{2}, \mathscr{O}(n-d)\right)$ with the line bundle $\mathscr{O}_{W}(-A)$. The Chern class of $E_{n} \otimes \mathscr{O}(A)$ is thus the Chern class of the direct image $\pi_{*}\left(\omega_{\mathscr{C} / W} \otimes \mathscr{L}^{n-d+3}\right)$; in particular we have

$$
c_{1}\left(E_{n}\right)=c_{1}\left(\pi_{*}\left(\omega_{\mathscr{G} / W} \otimes \mathscr{L}^{n-d+3}\right)\right)-\operatorname{rank}\left(E_{n}\right) \cdot A .
$$

Now suppose that $n \geq d-3$.In this case, the bundle $\omega_{\mathscr{C} / W} \otimes \mathscr{L}^{n-d+3}$ has no higher direct images (or, in the case $n=d-3$, a trivial one by relative duality), and the rank of $E_{n}$ is $(n+1)(n+2) / 2-\delta$. Setting $m=n-d+3$, by GrothendieckRiemann-Roch we have

$$
\begin{aligned}
c_{1}\left(\pi_{*}\right. & \left.\left(\omega_{\mathscr{K} / W} \otimes \mathscr{L}^{n-d+3}\right)\right) \\
& =\left[\pi_{*}\left(\operatorname{Td}(\mathscr{C} / W) \cdot \operatorname{ch}\left(\omega_{\mathscr{C} / W} \otimes \mathscr{L}^{m}\right)\right)\right]_{1} \\
& =\left[\pi_{*}\left(\left(1-\omega / 2+\left(\omega^{2}+\delta\right) / 12\right) \cdot\left(1+(\omega+m D)+(\omega+m D)^{2} / 2\right)\right)\right]_{1} \\
& =\pi_{*}\left((\omega+m D)^{2} / 2-(\omega+m D) \cdot \omega / 2+\left(\omega^{2}+\delta\right) / 12\right) \\
& =\left(m^{2} / 2\right) \cdot A+(m / 2) \cdot B+C / 12+\Delta / 12 .
\end{aligned}
$$

Thus

$$
c_{1}\left(E_{n}\right)=\left(m^{2} / 2-(n+1)(n+2) / 2+\delta\right) \cdot A+(m / 2) \cdot B+C / 12+\Delta / 12 .
$$

(b) Classes of the adjoint bundles via adjoint series. There is also an extrinsic approach to this computation. Suppose we have a flat family of zero-dimensional subschemes of $\mathbf{P}^{2}$ over a base $Z$-in other words, a subscheme $\Omega \subset \mathbf{P}^{2} \times Z$, flat over $Z$. let $\delta$ be the degree of $\Omega$ over $Z$, and let $D$ be the pullback to $\Omega$ of the class of line in $\mathbf{P}^{2}$. Suppose moreover that for all $z \in Z$ the fiber $\Omega_{Z} \subset \mathbf{P}^{2}$ imposes independent conditions on curves of degree $n$, and let $E_{n}$ be the vector bundle on $Z$ whose fiber at $z \in Z$ is the space of polynomials of degree $n$ vanishing on $\Omega_{Z}$. To determine the Chern class of the bundle $E_{n}$ let $\pi_{1}$ and $\pi_{2}$ denote the projection maps on $\mathbf{P}^{2} \times Z$, so that the exact sequence

$$
0 \rightarrow \mathscr{I}_{\Omega} \otimes \pi_{1}^{*} \mathscr{O}(n) \rightarrow \pi_{1}^{*} \mathscr{O}(n) \rightarrow \mathscr{O}_{\Omega} \otimes \pi_{1}^{*} \mathscr{O}(n) \rightarrow 0
$$

pushes forward to give an exact sequence

$$
0 \rightarrow E_{n} \rightarrow \mathscr{O}_{Z} \otimes H^{0}\left(\mathbf{P}^{2}, \mathscr{O}(n)\right) \rightarrow\left(\pi_{2}\right)_{*}\left(\mathscr{O}_{\Omega} \otimes \pi_{1}^{*} \mathscr{O}(n)\right) \rightarrow 0 .
$$


From this we may calculate

$$
\begin{aligned}
c_{1}\left(E_{n}\right) & =-c_{1}\left(\left(\pi_{2}\right)_{*}\left(\mathscr{O}_{\Omega} \otimes \pi_{1}^{*} \mathscr{O}(n)\right)\right) \\
& =-\pi_{2 *}\left(\operatorname{ch}\left(\mathscr{O}_{\Omega} \otimes \pi_{1}^{*} \mathscr{O}(n)\right) \cdot \operatorname{Td}(\Omega / Z)\right) \\
& =-\pi_{2 *}((\Omega \cdot n D)+[\mathrm{R}] / 2) \\
& =-n \cdot \pi_{2 *}(\Omega \cdot D)+\mathrm{Br} / 2
\end{aligned}
$$

where $\mathrm{R}=c_{1}\left(\omega_{\Omega / Z}\right)$ is the ramification divisor and $\mathrm{Br}$ the branch divisor, i.e., its image in $Z$. In our present circumstances, where $Z=W$ is the Severi variety and $\Omega=\bar{N} \subset \mathrm{P}^{2} \times Z$ the locus of assigned nodes, $\pi_{2 *}(\Omega \cdot D)=(2 d-3) A / 2-B / 2$, and we see by the local pictures of $S$ around cusps, tacnodes and triple points that

$$
B \sim T N+4 T R
$$

so that

$$
\begin{aligned}
c_{1}\left(E_{n}\right)= & -n((2 d-3) A / 2-B / 2)+T N / 2+2 T R \\
= & \frac{1}{4}(-2 n(2 d-3) A+2 n B+6(d-3) A+4(g-1) A \\
& +2(d-9) B-5 C+3 \Delta+4\left(d^{2}-6 d+8\right) A-8(g-1) A \\
& -4(d-6) B+16 C / 3-8 \Delta / 3) \\
= & \left(-n d+3 n / 2+d^{2}-9 d / 2+7 / 2-(g-1)\right) A \\
& +(n / 2-d / 2+3 / 2) B+C / 12+\Delta / 12,
\end{aligned}
$$

which agrees with our earlier computation, given the relation $g=(d-1)(d-2) / 2-\delta$.

Another way to interpret this approach is via the description given in the last chapter of the pullbacks of divisor classes from the Hilbert scheme $\mathscr{H}$ : it is well known (cf. [Ellingsrud-Stromme]) that the bundle $E_{n}$ on $\mathscr{H}$ has Chern class $-n \alpha+\beta / 2$; pulling back to $W$ and using formulas (4.25) we get the formula (5.3).

(c) The canonical class of the Severi variety. As we indicated, the bundle $E_{d}$ in particular can be related to the tangent bundle of $W$. In general, if $X \subset \mathbf{P}^{n}$ is any smooth $k$-dimensional subvariety of projective space, we have the associated projective Gauss map

$$
\gamma: X \rightarrow \mathbf{G}(k, n)
$$

sending each point $p \in X$ to the projective tangent plane $\gamma(p)=T_{p}(X) \cong \mathbf{P}^{k} \subset \mathbf{P}^{n}$. Let $S$ be the universal subbundle on the Grassmannian $\mathbf{G}(k, n)$; the Euler sequence

$$
0 \rightarrow \mathscr{O} \rightarrow \mathscr{O}(1)^{n+1} \rightarrow \Theta_{\mathbf{P}^{n}} \rightarrow 0
$$

for the tangent bundle to $\mathbf{P}^{n}$ pulls back to $X$ to give the sequence

$$
0 \rightarrow \mathscr{O} \rightarrow \gamma^{*} S \otimes \mathscr{O}(1) \rightarrow \Theta_{X} \rightarrow 0 .
$$

Observe that the definition of the Gauss map makes sense, and this last sequence holds, for any immersion $\rho: X \rightarrow \mathbf{P}^{n}$ of a smooth variety into projective space; we conclude in particular that the canonical class

$$
K_{X}=c_{1}\left(\gamma^{*} S^{*} \otimes \rho^{*} \mathscr{O}(-1)\right) .
$$

In our present circumstances, of course, the map from $W$ to $\mathbf{P}^{N}$ fails to be an immersion along the locus $C U$. The Gauss map is still regular, however, since the limiting position of the projective tangent plane $T_{C}(V)$ to the Severi variety 
$V \subset \mathbf{P}^{N}$ as $C$ approaches a cuspidal curve $C_{0}$ will always be the adjoint series of degree $d$; and the pullback $\gamma^{*} S$ of the universal subbundle is just the bundle $E_{d}$. The difference lies in the sequence (5.1): the pullback to $W$ of a regular vector field on $V$ may have a simple pole along $V$ in the direction of the (one-dimensional) kernel of $\rho_{*}$. Dually, the pullback map on 1-forms is not surjective, but maps into the subsheaf of $\Omega^{1}$ of forms vanishing on $\operatorname{ker}\left(\rho_{*}\right)$; since the cokernel of the map is a sheaf of rank 1 supported on $C U$ we have

$$
K_{W}=c_{1}\left(\gamma^{*} S^{*} \otimes \rho^{*} \mathscr{O}(-1)\right)+C U .
$$

In our present circumstance, the pullback $\gamma^{*} S$ is just the bundle $E_{d}$ introduced above, and by (5.1) this gives us

$$
\begin{aligned}
K_{W} & =-c_{1}\left(E_{d} \otimes \mathscr{O}_{W}(A)\right)+C U \\
& =-(9 / 2) \cdot A-(3 / 2) \cdot B-C / 12-\Delta / 12+C U \\
& =-(3 / 2) A+(3 / 2) B+(11 / 12) C-(13 / 12) \Delta .
\end{aligned}
$$

\section{REFERENCES}

[ACGH] E. Arbarello, M. Cornalba, P. Griffiths and J. Harris, Geometry of algebraic curves, Springer-Verlag, New York, 1984.

[Cukierman] F. Cukierman, Ph.D. thesis, Brown University, 1987.

[Diaz-Harris] S. Diaz and J. Harris, Ideals associated to deformations of singular plane curves, Trans. Amer. Math. Soc. (to appear)

[Eisenbud-Harris 1] D. Eisenbud and J. Harris, When ramification points meet, Invent. Math. 87 (1987), 485-493.

[Eisenbud-Harris 2] D. Eisenbud and J. Harris, Limit limit series: basic theory, Invent. Math. 85 (1986), 337-371.

[Ellingsrud-Stromme] G. Ellingrud and S. Stromme, On the homology of the Hilbert scheme of points in the plane, preprint no. 13, Univ. of Oslo, 1984.

[Fulton] W. Fulton, On nodal curves, Algebraic Geometry: Open Problems, Lecture Notes in Math., vol. 997 Springer-Verlag, Berlin, 1983, pp. 146-155.

[Fulton, book] W. Fulton, Intersection theory, Springer-Verlag, Berlin, 1984.

[Harris] J. Harris, On the Severi problem, Invent. Math. 84 (1986), 445-461.

[Harris, AMS notes] J. Harris, Curves and their moduli, Algebraic Geometry-Bowdoin 1985, Proc. Sympos. Pure Math., vol. 46, Amer. Math. Soc., Providence, R.I., 1987, pp. 99-143.

[Ran] Z. Ran, Degenerations of linear systems, preprint.

Department of Mathematics, University of Pennsylvania, Philadelphia, PENNSYLVANIA 19104 (Current address of Steven Diaz)

Department of Mathematics, Brown University, Providence, Rhode Island 02912

Current address (Joe Harris): Department of Mathematics, Harvard University, Cambridge, Massachusetts 02138 